\title{
Article \\ Synthesis of 3,4-Dihydropyrimidin-2(1H)-one-phosphonates by the Microwave-Assisted Biginelli Reaction
}

\author{
Nóra Popovics-Tóth, Ádám Tajti $\mathbb{1}$, Evelyn Hümpfner and Erika Bálint * \\ Department of Organic Chemistry and Technology, Budapest University of Technology and Economics, \\ 1521 Budapest, Hungary; toth.nora@mail.bme.hu (N.P.-T.); tajti.adam@mail.bme.hu (Á.T.); \\ h.eve95@gmail.com (E.H.) \\ * Correspondence: balint.erika@vbk.bme.hu; Tel.: +36-1-463-3653
}

Citation: Popovics-Tóth, N.; Tajti, Á.; Hümpfner, E.; Bálint, E. Synthesis of 3,4-Dihydropyrimidin-2(1H)-OnePhosphonates by the MicrowaveAssisted Biginelli Reaction. Catalysts 2021, 11, 45. https://doi.org/ $10.3390 /$ catal11010045

Received: 15 December 2020 Accepted: 29 December 2020 Published: 31 December 2020

Publisher's Note: MDPI stays neutral with regard to jurisdictional clai$\mathrm{ms}$ in published maps and institutional affiliations.

Copyright: (C) 2020 by the authors. Licensee MDPI, Basel, Switzerland. This article is an open access article distributed under the terms and conditions of the Creative Commons Attribution (CC BY) license (https:// creativecommons.org/licenses/by/ $4.0 /)$.
Abstract: The synthesis of novel 3,4-dihydropyrimidin-2(1H)-one-phosphonates was elaborated by the microwave (MW)-assisted three-component Biginelli reaction of $\beta$-ketophosphonates, aromatic or aliphatic aldehydes and urea derivatives. The condensation was optimized on a selected model reaction in respect of the reaction parameters, such as the heating method, the type of the catalyst and solvent, the temperature, the reaction time and the molar ratio of the starting materials. The fast and solvent-free MW-assisted procedure was then extended for the preparation of further new 3,4-dihydropyrimidin-2(1H)-one-phosphonate derivatives starting from different aromatic aldehydes, $\beta$-ketophosphonates and urea derivatives to prove the wide scope of the process. As a novel by-product of the Biginelli-type synthesis of 3,4-dihydropyrimidin-2(1H)-one-phosphonates, the 5-diethoxyphosphoryl-4-phenyl-6-styryl-3,4-dihydropyrimidin-2 $(1 H)$-one was also isolated and characterized. Our MW-assisted method made also possible the condensation of aliphatic aldehydes, diethyl (2-oxopropyl)phosphonate and urea, which reaction was previously reported to be impossible in the literature.

Keywords: heterocyclic phosphonates; 3,4-dihydropyrimidin-2(1H)-one-phosphonates; multicomponent reactions; Biginelli reaction; microwave

\section{Introduction}

A wide number of organophosphorus compounds play an important role in different fields of life; in organic- and medicinal chemistry, as well as in the agriculture and plastic industries [1]. Organic phosphonates may be considered as one of the most important subgroups. Among them, $\alpha$-aminophosphonates are the bioisosteres of natural $\alpha$-amino acids, and due to this structural similarity, they were proved to be effective as antibiotics, antiviral, or antitumor agents, as well as pesticides [2-6]. Over the last decade, due to their structural diversity, heterocyclic phosphonates have received an intensively growing interest $[7,8]$. A few biologically active examples can be seen in Figure 1. $\alpha$ Aminophosphonates containing a piperidine ring (1) showed activity as an insecticide against Plutella xylostella [9]. A few 1,2,3-triazol-5-yl-phosphonates (2) were identified as promising cytotoxic and antibacterial agents [10]. Similarly, to the 1,4-dihydropyridine3,5-dicarboxylates, several 1,4-dihydropyridine-5-phosphonates (3) showed significant antihypertensive activity [11-13].

Over the last decades, a number of biologically active 3,4-dihydropyrimidin-2(1H)-one carboxylates were utilized as anticancer-, antihypertensive-, anti-inflammatory-, antibacterial-, antiviral- or antifugal agents (Figure 2) [14,15]. Monastrol (4), a cell-permeable small molecule inhibitor, was introduced in 1999 [16]. Another important derivative is Piperastrol (5), which was proved to be effective against cancer cell lines [17]. As for the closely related 3,4-dihydropyrimidin-2(1H)-one phosphonate derivatives (6), the anti-inflammatory effect was investigated [18]. 
<smiles>[R]c1ccc(NC2(P(=O)(O)OCC)CCN(S(=O)(=O)c3ccc(C)cc3)CC2)cc1</smiles>

$\mathrm{R}=\mathrm{H}, 2-\mathrm{C}, 3-\mathrm{Cl}, 4-\mathrm{Cl}, 2-\mathrm{F}$,

3-F, 4-F, 2-Me, 2-NO

1
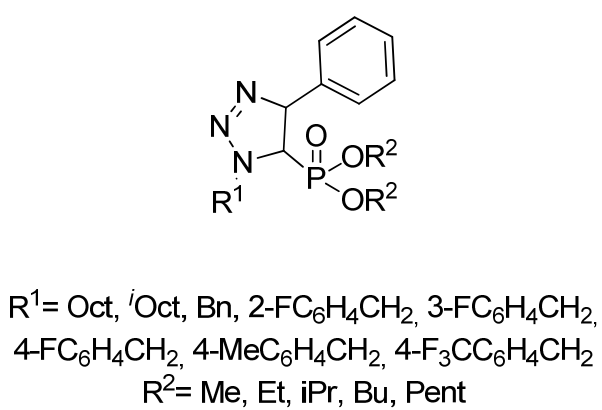

2<smiles>[R]OC(=O)C1=C(C)NC(C)=C(P(=O)(OC)OCC([R])([R])CO)C1c1[R]cccc1</smiles>

$\mathrm{R}^{1}=2-\mathrm{NO}_{2}, 2-\mathrm{Cl}, 3-\mathrm{Cl}, 2-\mathrm{CF}_{3}, 2-\mathrm{OCHF}_{2}$

$\mathrm{R}^{2}=\mathrm{Me},{ }^{i} \mathrm{Pr},{ }^{n} \mathrm{Hex}$

$\mathrm{R}^{3}=\mathrm{H}, \mathrm{Me}$

3

Figure 1. Biologically active heterocyclic phosphonates.<smiles>CCOC(=O)C1=C(C)NC(=S)NC1c1cccc(O)c1</smiles>

4<smiles>CCOC(=O)C1=C(C)NC(=S)NC1c1ccc2c(c1)OCO2</smiles>

5<smiles>[R][R]P([R20])(=O)C1=C(C)NC(=O)NC1c1ccccc1</smiles>

$\mathrm{R}^{1}=\mathrm{Me}, \mathrm{Et}$

$\mathrm{R}^{2}=\mathrm{H}, 4-\mathrm{NO}_{2}, 4-\mathrm{Cl}, 4-\mathrm{MeO}, 4-\mathrm{Me}$

Figure 2. 3,4-Dihydropyrimidin-2(1H)-one derivatives.

One of the most useful tools for the preparation of heterocyclic phosphonates is their synthesis via multicomponent reactions (MCR) [19]. These transformations have several benefits, such as the high atom economy, the fast and simple accomplishment, and the ability to save time and energy [20]. In addition, they usually mean a suitable way for creating large molecular libraries. The efficiency of the MCRs can be further improved by the microwave (MW) technique [21,22]. In most cases, applying MW irradiation, the reactions are faster and more selective, and it allows to reach higher yields as compared to the conventionally heated experiments $[23,24]$. Moreover, it is usually suitable for carrying out solvent- and/or catalyst-free reactions. Due to these advantages, MW-assisted MCRs may be ideal for the rapid and efficient synthesis of new chemical libraries.

One of the well-known examples of MCRs is the three-component Biginelli reaction, in which a $\beta$-ketocarboxilic ester (7), an aldehyde and a urea derivative react in a one-pot manner to form dihydropyrimidin-2(1H)-ones (8) (Scheme 1).<smiles>[R]C=[O+]C(=O)CC([R])=O</smiles>

7<smiles>[R]NC([X])N</smiles>

$\mathrm{R}^{4}$<smiles>CCC</smiles><smiles>[R]OC(=O)C1=C([R])N([R])C([X])NC1[R]</smiles>

8

Scheme 1. Biginelli reaction of $\beta$-ketocarboxilic esters, aldehydes, and urea derivatives.

Although the Biginelli reaction of regular $\beta$-ketocarboxilic acid esters is a widely investigated process $[25,26]$, there are only a few examples for the condensation of $\beta$ ketophosphonates $[18,27,28]$. In order to compare the reactivity of the two different $\mathrm{CH}$-acidic moieties, Chebil and co-workers studied the reaction of $\beta$-keto- $\alpha$-carbethoxyphosphonate derivatives (9), aldehydes and urea in the presence of acetic acid in ethanol (Scheme 2) [29]. 
It was found that only the carboxyl $\mathrm{CH}$-acidic function was reactive, resulting in 5carbethoxy-6-phosphonomethyl-3,4-dihidropyrimidin-2(1H)-ones (10) as the products. These experiments predicted a significant reactivity difference between $\beta$-ketophosphonates and $\beta$-ketocarboxylic esters.
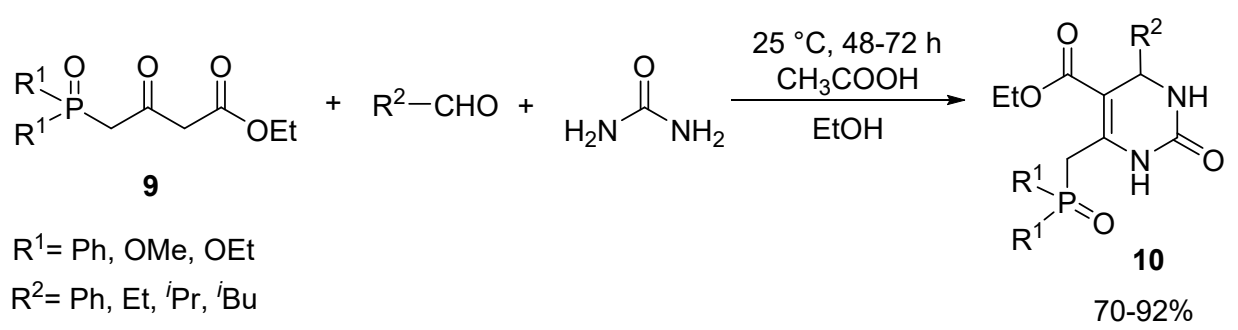

Scheme 2. Condensation of $\beta$-keto- $\alpha$-carbethoxyphosphonates (9), aldehydes and urea.

In the literature, only three examples were reported on the Biginelli reaction of $\beta$ ketophosphonates. The condensation of diethyl or dimethyl (2-oxopropyl)phosphonate, aromatic aldehydes and urea was carried out in the presence of $50 \mathrm{~mol} \%$ of $p$-toluene sulfonic acid (PTSA) in acetonitrile [27], $5 \mathrm{~mol} \%$ of $\mathrm{Yb}(\mathrm{OTf})_{3}$ in toluene [28], or $15 \mathrm{~mol} \%$ of $\mathrm{Zn}(\mathrm{OTf})_{2}$ in toluene [18]. In all cases, urea was used in excess of 1.5 equivalents. The current literature methods only enable the synthesis of dihydropyrimidin-2(1H)-one phosphonate derivatives applying long reaction times (3-24 h) and a solvent. It should also be noted that a comprehensive study on the reaction parameters is still missing.

Based on the literature data, aliphatic aldehydes were found to be inactive in the threecomponent reaction $[27,28]$. The condensation of (2-oxopropyl)phosphonate and urea was carried out with propionaldehyde or butyraldehyde, and no product was detected [28].

In this paper, our aim was to study and optimize the Biginelli reaction of diethyl (2-oxopropyl)phosphonate, benzaldehyde and urea in respect of the reaction conditions (e.g., the heating method, the catalyst type, the solvent type, the temperature, the reaction time and the molar ratio of the starting materials). Other goals were to extend the synthesis for further $\beta$-ketophosphonates, aromatic aldehydes and urea derivatives, and the characterization on the novel dihydropyrimidin- $2(1 H)$-one phosphonates by NMR spectroscopy and HRMS. We also aimed at elaborating the analogous condensation starting from aliphatic aldehydes as novel substrates.

\section{Results and Discussion}

At first, the model reaction of diethyl (2-oxopropyl)phosphonate, benzaldehyde and urea was investigated (Table 1). The condensation was followed by ${ }^{31} \mathrm{P}$ NMR spectroscopy, the crude products were analyzed by High Performance Liquid Chromatography Mass Spectrometry (HPLC-MS). The first experiments were performed reacting the three components at a molar ratio of 1:1:1.5 in an oil bath at the boiling point of acetonitrile (MeCN) $\left(82{ }^{\circ} \mathrm{C}\right.$ ) for $4 \mathrm{~h}$ (Table $1 /$ Entries 1-6). Applying $50 \mathrm{~mol} \%$ of PTSA as a catalyst, only $9 \%$ of the desired 5-diethoxyphosphoryl-6-methyl-4-phenyl-3,4-dihydropyrimidin-2(1H)-one (11a) was obtained, and a by-product containing a styryl group at the position of six (12a) was detected in $2 \%$ (Table 1/Entry 1 ). The by-product formation will be discussed later in details. In the next series of experiments, $15 \mathrm{~mol} \%$ of scandium-, ytterbium- or zinc triflate was tried out as a catalyst (Table 1/Entries 2-4). Among them, $\mathrm{Zn}(\mathrm{OTf})_{2}$ was the most efficient, the proportion of the target compound (11a) was $45 \%$ (Table 1/Entry 4). In case of $\mathrm{Sc}(\mathrm{OTf})_{3}$ and $\mathrm{Yb}(\mathrm{OTf})_{3}, 5 \%$ and $24 \%$ of the dihydropyrimidin-2(1H)-one (11a) was formed, respectively (Table $1 /$ Entries 2 and 3). After that, the effect of the $\mathrm{Zn}(\mathrm{OTf})_{2}$ amount was investigated (Table 1/Entries 2, 5 and 6). Carrying out the reaction in the presence of $10 \mathrm{~mol} \%$ of $\mathrm{Zn}(\mathrm{OTf})_{2}$, the conversion was only $35 \%$ (Table $1 /$ Entry 5 ), while $20 \mathrm{~mol} \%$ of the catalyst did not change the composition as compared to the condensation catalyzed by $15 \mathrm{~mol} \%$ of $\mathrm{Zn}(\mathrm{OTf})_{2}$ (Table $1 /$ Entry 6 ). Then, the model reaction was studied at a higher temperature of $100{ }^{\circ} \mathrm{C}$ (Table $1 /$ Entry 7 ). After $3 \mathrm{~h}, 47 \%$ of product $11 \mathrm{a}$ and $10 \%$ 
of by-product 12a were formed. Performing the condensation in toluene (PhMe) under the same conditions, the ratio of product 11a and by-product 12a increased to $52 \%$ and $16 \%$, respectively (Table 1/Entry 8). A solvent-free variation was also carried out at $100{ }^{\circ} \mathrm{C}$ for $3 \mathrm{~h}$, when $27 \%$ of diethyl (2-oxopropyl)phosphonate (A), 59\% of dihydropyrimidin-2(1H)-one (11a) and 14\% of 5-diethoxyphosphoryl-4-phenyl-6-styryl-3,4-dihydropyrimidin-2(1H)-one (12a) were present in the reaction mixture (Table 1/Entry 9). Based on the results, the reaction was more efficient in the absence of solvent. After that, the three-component reaction was studied under MW irradiation (Table 1/Entries 9-16). Performing the condensation in the presence of $15 \mathrm{~mol} \%$ of $\mathrm{Zn}(\mathrm{OTf})_{2}$ at $100{ }^{\circ} \mathrm{C}$ for $2 \mathrm{~h}$ without any solvent in a $\mathrm{MW}$ reactor, the conversion was already $72 \%$, and the mixture comprised $66 \%$ of product 11 a and $6 \%$ of by-product 12a (Table 1/Entry 10). In order to study the influence of the MW irradiation, the reaction was also carried out in the absence of any catalyst, and the formation of 33\% of product 11a was observed (Table 1/Entry 11). However, the catalyst-free MW-assisted variation did not reach the conversion value of the catalyzed reaction, the influence of the MW irradiation was significant, which also confirms the efficiency of the MW heating for MCRs as described in the Literature part. Increasing the reaction time from $2 \mathrm{~h}$ to $4 \mathrm{~h}$, and using $15 \mathrm{~mol} \%$ of $\mathrm{Zn}(\mathrm{OTf})_{2}$, the proportion of the desired compound (11a) $(66 \%$ and $71 \%$, respectively) did not increase significantly (Table 1/Entry 10 and Entry 12). After that, the molar ratio of the starting materials was optimized (Table 1/Entry 13-16). Applying 2 equivalents of urea, the ratio of the dihydropyrimidin-2(1H)-one phosphonate (11a) increased to $73 \%$ (Table 1/Entry 13), while with 2.5 equivalents of urea, the composition remained almost the same $(74 \%)$ (Table $1 /$ Entry 14$)$. Then the molar ratio of benzaldehyde was increased using 2 equivalents of the urea (Table 1/Entries 15 and 16). Carrying out the reaction with 1.2 equivalents of benzaldehyde, the conversion was almost complete, and the proportion of the target product (11a) was $82 \%$ (Table 1/Entry 15). Using 1.5 equivalents of benzaldehyde, a conversion of $100 \%$ was achieved (Table 1/Entry 16), and 89\% of product 11a and 11\% of by-product 12a were formed. The desired dihydropyrimidin$2(1 H)$-one phosphonate (11a) was isolated in a yield of $75 \%$ after column chromatography. The optimized conditions include using 1.5 equivalents of benzaldehyde and 2 equivalents of urea in the presence of $15 \mathrm{~mol} \%$ of $\mathrm{Zn}(\mathrm{OTf})_{2}$ at $100^{\circ} \mathrm{C}$ for $2 \mathrm{~h}$ under solvent-free $\mathrm{MW}$ conditions (Table 1/Entry 16).

In most of the experiments, formation of the 5-diethoxyphosphoryl-4-phenyl-6-styryl3,4-dihydropyrimidin-2(1H)-one (12a) was observed, which can probably be considered as a by-product of the Biginelli reaction of $\beta$-ketophosphonates, however, it was not known in the literature. The mechanism of its formation may be similar to the by-product formation in the regular Biginelli condensation reported by Zang and co-workers [30]. The proposed mechanism of the formation can be seen in Scheme 3. As the first step, the $\mathrm{Zn}(\mathrm{OTf})_{2}$ catalyst initiates the formation of intermediate I, which goes into an aldol condensation with the remaining benzaldehyde in the reaction mixture to form intermediate II. After losing a water molecule from intermediate II, the 5-diethoxyphosphoryl-4-phenyl-6-styryl3,4-dihydropyrimidin-2(1H)-one (12a) by-product is formed. 
Table 1. Biginelli reaction of diethyl (2-oxopropyl)phosphonate, benzaldehyde and urea.

\begin{tabular}{|c|c|c|c|c|c|c|c|c|c|}
\hline & $\mathbf{A}^{\mathrm{OE}}$ & B & ${ }_{\mathbf{C}}{ }_{\mathrm{H}}^{\mathrm{O}}$ & $\begin{array}{r}\Delta \text { or } \mathrm{N} \\
\mathrm{T}, \mathrm{t} \\
\text { catal }\end{array}$ & & $11 a$ & & $12 a$ & \\
\hline \multirow{2}{*}{ Entry } & \multirow{2}{*}{$\begin{array}{l}\text { Mode of } \\
\text { Heating }\end{array}$} & \multirow{2}{*}{$\begin{array}{c}\text { A:B:C } \\
\text { [equiv.] }\end{array}$} & \multirow{2}{*}{ Catalyst } & \multirow{2}{*}{ Solvent } & \multirow{2}{*}{$\begin{array}{c}\mathbf{T} \\
{\left[{ }^{\circ} \mathrm{C}\right]}\end{array}$} & \multirow{2}{*}{$\begin{array}{c}t \\
{[h]}\end{array}$} & \multicolumn{3}{|c|}{ Composition $[\%]^{a}$} \\
\hline & & & & & & & $\mathbf{A}$ & 11a & $12 \mathrm{a}$ \\
\hline 1 & $\Delta$ & 1:1:1.5 & $50 \%$ PTSA & $\mathrm{MeCN}$ & 82 & 4 & 89 & 9 & 2 \\
\hline 2 & $\Delta$ & 1:1:1.5 & $15 \% \mathrm{Sc}(\mathrm{OTf})_{3}$ & $\mathrm{MeCN}$ & 82 & 4 & 95 & 5 & 0 \\
\hline 3 & $\Delta$ & $1: 1: 1.5$ & $15 \% \mathrm{Yb}(\mathrm{OTf})_{3}$ & $\mathrm{MeCN}$ & 82 & 4 & 76 & 24 & 0 \\
\hline 4 & $\Delta$ & $1: 1: 1.5$ & $15 \% \mathrm{Zn}(\mathrm{OTf})_{2}$ & $\mathrm{MeCN}$ & 82 & 4 & 54 & 45 & 1 \\
\hline 5 & $\Delta$ & 1:1:1.5 & $10 \% \mathrm{Zn}(\mathrm{OTf})_{2}$ & $\mathrm{MeCN}$ & 82 & 4 & 65 & 35 & 0 \\
\hline 6 & $\Delta$ & $1: 1: 1.5$ & $20 \% \mathrm{Zn}(\mathrm{OTf})_{2}$ & $\mathrm{MeCN}$ & 82 & 4 & 54 & 45 & 1 \\
\hline 7 & $\Delta$ & 1:1:1.5 & $15 \% \mathrm{Zn}(\mathrm{OTf})_{2}$ & $\mathrm{MeCN}$ & 100 & 3 & 43 & 47 & 10 \\
\hline 8 & $\Delta$ & 1:1:1.5 & $15 \% \mathrm{Zn}(\mathrm{OTf})_{2}$ & $\mathrm{PhMe}$ & 100 & 3 & 32 & 52 & 16 \\
\hline 9 & $\Delta$ & 1:1:1.5 & $15 \% \mathrm{Zn}(\mathrm{OTf})_{2}$ & - & 100 & 3 & 27 & 59 & 14 \\
\hline 10 & MW & $1: 1: 1.5$ & $15 \% \mathrm{Zn}(\mathrm{OTf})_{2}$ & - & 100 & 2 & 28 & 66 & 6 \\
\hline 11 & MW & $1: 1: 1.5$ & - & - & 100 & 2 & 55 & 33 & 12 \\
\hline 12 & MW & $1: 1: 1.5$ & $15 \% \mathrm{Zn}(\mathrm{OTf})_{2}$ & - & 100 & 4 & 24 & 71 & 5 \\
\hline 13 & MW & $1: 1: 2$ & $15 \% \mathrm{Zn}(\mathrm{OTf})_{2}$ & - & 100 & 2 & 23 & 73 & 4 \\
\hline 14 & MW & $1: 1: 2.5$ & $15 \% \mathrm{Zn}(\mathrm{OTf})_{2}$ & - & 100 & 2 & 21 & 74 & 5 \\
\hline 15 & MW & 1:1.2:2 & $15 \% \mathrm{Zn}(\mathrm{OTf})_{2}$ & - & 100 & 2 & 7 & 84 & 9 \\
\hline 16 & MW & $1: 1.5: 2$ & $15 \% \mathrm{Zn}(\mathrm{OTf})_{2}$ & - & 100 & 2 & 0 & $89^{b}$ & 11 \\
\hline
\end{tabular}

${ }^{\mathrm{a}}$ Based on ${ }^{31} \mathrm{P}$ NMR. ${ }^{\mathrm{b}}$ An isolated yield of $75 \%$ was obtained.

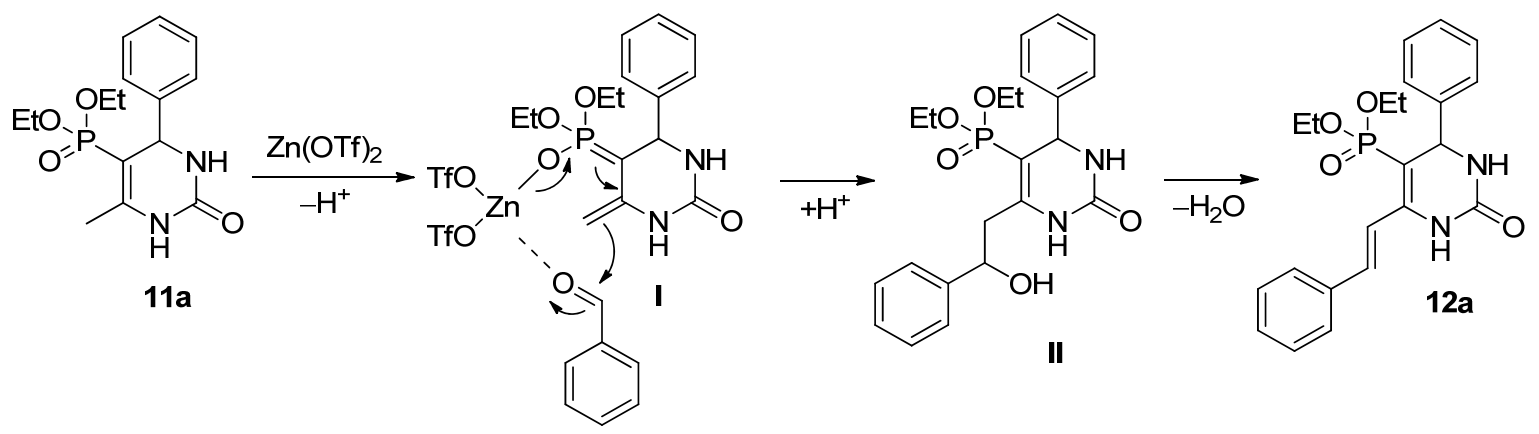

Scheme 3. Proposed mechanism of the formation of compound 12a.

As a structure proving synthesis, 5-diethoxyphosphoryl-6-methyl-4-phenyl-3,4dihydropyrimidin-2(1H)-one (11a) was reacted with 2 equivalents of benzaldehyde in acetonitrile at $82{ }^{\circ} \mathrm{C}$ for $24 \mathrm{~h}$ in the presence of $10 \mathrm{~mol} \%$ of $\mathrm{Zn}(\mathrm{OTf})_{2}$ (Scheme 4). The 5-diethoxyphosphoryl-4-phenyl-6-styryl-3,4-dihydropyrimidin-2(1H)-one (12a) could be isolated from the reaction mixture, and it was characterized by ${ }^{31} \mathrm{P},{ }^{1} \mathrm{H}$ and ${ }^{13} \mathrm{C}$ NMR spectroscopy, as well as HRMS. The analytical data were identical to the observed signals of the by-product during the Biginelli reactions. 
<smiles>CCOP(=O)(OCC)C1=C(C)NC(=O)NC1c1ccccc1C1NC(=O)NC(/C=C/c2ccccc2)=C1P(=O)(OCC)OCC</smiles>

Scheme 4. Structure proving synthesis of by-product 12a.

Next, the MW-assisted Biginelli reaction was extended to various $\beta$-ketophosphonates (diethyl and dimethyl (2-oxopropyl)phosphonate) and to a series of aromatic aldehydes (benzaldehyde, 2-chlorobenzaldehyde, 3-chlorobenzaldehyde, 4-chlorobenzaldehyde, 4fluorobenzaldehyde, 2-fluoro-4-iodobenzaldehyde, 3-methylbenzaldehyde, 4-hydroxybenzaldehyde, 4-nitrobenzaldehyde, 3,4,5-trimethoxybenzaldehyde) (Scheme 5). The solventfree condensations were performed applying the optimized parameters $\left(15 \mathrm{~mol} \% \mathrm{Zn}(\mathrm{OTf})_{2}\right.$, $100{ }^{\circ} \mathrm{C}, 2 \mathrm{~h}$ ) under MW conditions. Overall, 20 dihydropyrimidin-2(1H)-one phosphonate derivatives (11a-j and 13a-j) were synthesized in yields of 56-81\% after column chromatography, from which 14 derivatives $(\mathbf{1 1 b}, \mathbf{1 1}$, $\mathbf{1 1 e}-\mathbf{h}, \mathbf{1 1} \mathbf{j}, \mathbf{1 3 b}, \mathbf{1 3 c}, \mathbf{1 3 e}-\mathbf{h}$ and $\mathbf{1 3} \mathbf{j})$ are new compounds. Although the yields of the Biginelli reaction were slightly influenced by the reactivity of the different aromatic aldehydes, the outcome was almost independent from the starting $\beta$-ketophosphonate.

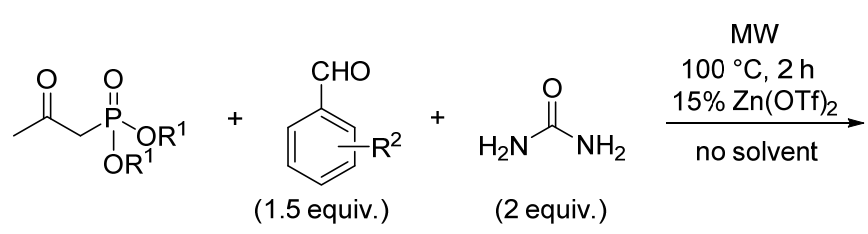<smiles>[R][R]P([R20])(=O)C1=C(C)NC(=O)NC1c1ccccc1</smiles>

$11\left(R^{1}=\mathrm{Et}\right)$ or $13\left(R^{1}=\mathrm{Me}\right)$

$\mathrm{R}^{2}=\mathrm{H}(\mathbf{a}), 2-\mathrm{Cl}(\mathbf{b}), 3-\mathrm{Cl}(\mathbf{c}), 4-\mathrm{Cl}(\mathbf{d})$,

4-F (e), 2-F-4-I (f), 3-Me (g), 4-OH (h),

$\mathrm{NO}_{2}$ (i), 3,4,5-tri(MeO) (j)<smiles>[R20]P([R20])(=O)C1=C(C)NC(=O)NC1c1ccccc1</smiles>

11a: $75 \%^{a}$ 13a: $80 \%^{a}$<smiles>[R20]P([R20])(=O)C1=C(C)NC(=O)NC1c1ccc(I)cc1F</smiles>

11f: $61 \%^{\mathrm{a}}$

13f: $63 \%^{a}$<smiles>[R20]P([R20])(=O)C1=C(C)NC(=O)NC1c1ccccc1Cl</smiles>

11b: $66 \%$ a

13b: $62 \%^{a}$<smiles>[R2][R]([R2])([H])C1=C(C)NC(=O)NC1c1cccc(Cl)c1</smiles>

11c: $75 \%^{a}$

13c: $75 \%^{a}$<smiles>[R7][R]([R2])([H])C1=C(C)NC(=O)NC1c1cccc([N+](=O)[O-])c1</smiles>

11g: $72 \%^{a}$

13g: $76 \%^{\mathrm{a}}$<smiles>[R2][R]([R2])([H])C1=C(C)NC(=O)NC1c1ccc(O)cc1</smiles>

11h: $62 \%^{\mathrm{a}}$

13h: $60 \%^{\mathrm{a}}$<smiles>[R20]P([R20])(=O)C1=C(C)NC(=O)NC1c1ccc(Cl)cc1</smiles>

13d: $67 \%^{\mathrm{a}}$<smiles>[R7][R]([R2])([H])C1=C(C)NC(=O)NC1c1ccc([N+](=O)[O-])cc1</smiles><smiles>[R7]P([R2])(=O)C1=C(C)NC(=O)NC1c1ccc(F)cc1</smiles>

11e: $79 \%^{\mathrm{a}}$

13e: $81 \%^{\mathrm{a}}$<smiles>[R7]P([R2])(=O)C1=C(C)NC(=O)NC1c1cc(OC)c(OC)c(OC)c1</smiles>

11j: $60 \%^{\mathrm{a}}$

13j: $56 \%^{\mathrm{a}}$

a Isolated yield.

Scheme 5. Biginelli reaction of diethyl or dimethyl (2-oxopropyl)phosphonate, substituted benzaldehydes and urea. 
The Biginelli reaction of $\beta$-ketophosphonates (diethyl and dimethyl (2-oxopropyl) phosphonate) was also investigated with benzaldehyde and $N$-methylurea (Scheme 6). Applying the optimal conditions, the new $N$-Me-dihydropyrimidin-2(1H)-one-phosphonates (14a and 14b) could be synthesized in yields of 56\% and 53\%, respectively.

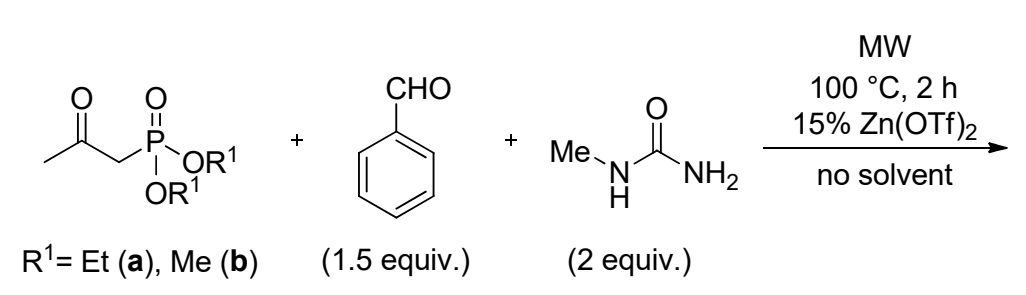

a Isolated yield.<smiles>[Y6]N1C(=O)NC(c2ccccc2)C(P([R20])(=O)O)=C1C</smiles>

14a: $56 \%^{a}$

14b: $53 \%^{a}$

Scheme 6. Biginelli reaction of $\beta$-ketophosphonates, benzaldehyde and $N$-methylurea.

According to literature reports, aliphatic aldehydes are not suitable substrates in the Biginelli reaction starting from $\beta$-ketophosphonates $[27,28]$. However, one of our aims was to accomplish the condensation with aliphatic aldehydes as well (Scheme 7). At first, the reaction of diethyl (2-oxopropyl)phosphonate, 1.5 equivalents of butyraldehyde and 2 equivalents of urea was carried out in the absence of any solvent applying the optimized conditions (15 mol\% $\left.\mathrm{Zn}(\mathrm{OTf})_{2}, 100{ }^{\circ} \mathrm{C}, 2 \mathrm{~h}\right)$ under MW irradiation. The 4-propyldihydropyrimidin-2(1H)-one phosphonate (15a) could be synthesized in a yield of $41 \%$ (Scheme 6). Then, the condensation was extended to dimethyl (2-oxopropyl)phosphonate and isovaleraldehyde as well. Utilizing the MW-assisted synthesis developed, four novel 4-alkyl-dihydropyrimidin-2(1H)-one phosphonates $\mathbf{( 1 5} \mathbf{a}, \mathbf{b}$ and $\mathbf{1 6} \mathbf{a}, \mathbf{b})$ were prepared in yields of $41-43 \%$.

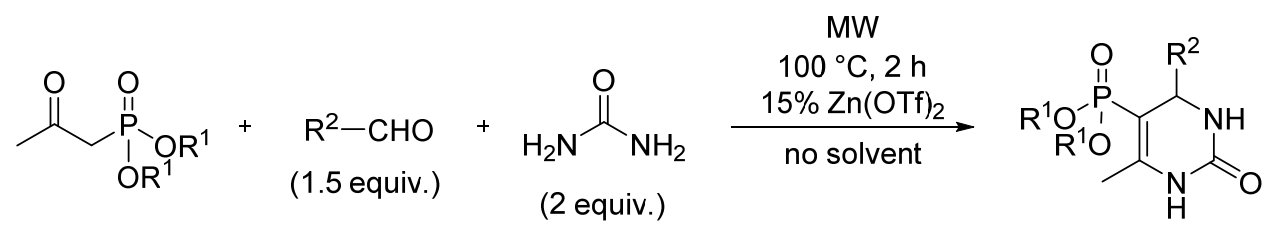<smiles>CCCC1NC(=O)NC(C)=C1P(=O)(OCC)OCC</smiles><smiles>CCCC1NC(=O)NC(C)=C1P(=O)(OC)OC</smiles><smiles>CCOP(=O)(OCC)C1=C(C)NC(=O)NC1CC(C)C</smiles><smiles>COP(=O)(OC)C1=C(C)NC(=O)NC1CC(C)C</smiles>

a Isolated yield.

Scheme 7. Biginelli reaction of diethyl or diethyl (2-oxopropyl)phosphonate, aliphatic aldehydes and urea.

The MW-assisted approach developed is an efficient and simple methodology for the synthesis of 3,4-dihydropyrimidin-2(1H)-one-phosphonates by the Biginelli reaction of $\beta$ ketophosphonates, which apply milder, faster, and greener reaction conditions compared to the previous reports $[18,27,28]$. Besides a comprehensive optimization, we have provided 
exact product compositions, including the by-product containing a styryl group at the position of $\operatorname{six}(\mathbf{1 2 a})$.

\section{Materials and Methods}

\subsection{General}

All chemicals were acquired from Fluorochem Ltd., Hadfield, UK and used without further purification, e.g., diethyl (2-oxopropyl)phosphonate (95\% purity), dimethyl (2oxopropyl)phosphonate ( $97 \%$ purity), urea ( $>99 \%$ purity), benzaldehyde (>98\% purity), zinc triflate (anhydrous, 98\% purity).

The reactions under conventional heating were carried out in an oil bath. The microwave-assisted experiments were performed in a $300 \mathrm{~W} \mathrm{CEM}^{\circledR}$ Discover $^{\circledR}$ focused microwave reactor (CEM Microwave Technology Ltd., Buckingham, UK) equipped with a pressure controller using 5-20 W irradiation under isothermal conditions.

HPLC-MS measurements were performed with an Agilent 1200 liquid chromatography system coupled with a 6130 quadrupole mass spectrometer equipped with an ESI ion source (Agilent Technologies, Palo Alto, CA, USA). Analysis was performed at $40{ }^{\circ} \mathrm{C}$ on a Gemini C18 column (150 mm $\times 4.6 \mathrm{~mm}, 3 \mu \mathrm{m}$; Phenomenex, Torrance, CA, USA) with a mobile phase flow rate of $0.6 \mathrm{~mL} / \mathrm{min}$. Composition of eluent $\mathrm{A}$ was $0.1 \%\left(\mathrm{NH}_{4}\right)(\mathrm{HCOO})$ in water; eluent $\mathrm{B}$ was $0.1 \%\left(\mathrm{NH}_{4}\right)(\mathrm{HCOO})$ and $8 \%$ water in acetonitrile. $0-3 \mathrm{~min} .5 \% \mathrm{~B}, 3-13$ min. gradient, 13-20 min. 100\% B. The injection volume was $5 \mu \mathrm{L}$. The chromatographic profile was registered at $222 \mathrm{~nm}$. TheMSD operating parameters were as follows: positive ionization mode, scan spectra from m/z 120 to 1200 , drying gas temperature $300{ }^{\circ} \mathrm{C}$, nitrogen flow rate $10 \mathrm{~L} / \mathrm{min}$, nebulizer pressure 60 psi, capillary voltage $4000 \mathrm{~V}$.

High resolution mass spectrometric measurements were performed using a Sciex $5600+$ Q-TOF mass spectrometer (AB Sciex UK Limited, Warrington, UK) in positive electrospray mode.

The ${ }^{31} \mathrm{P},{ }^{1} \mathrm{H},{ }^{13} \mathrm{C}$, NMR spectra (see in Supplementary Materials) were taken in DMSO$\mathrm{D}_{6}$ solution on a Bruker AV-300 spectrometer (Bruker Scientific LLC, Billerica, MA, USA) operating at 121.5, 300 and $75.5 \mathrm{MHz}$, respectively. Chemical shifts are downfield relative to $85 \% \mathrm{H}_{3} \mathrm{PO}_{4}$ and TMS. Non-equivalence effects were observed in the ${ }^{1} \mathrm{H}$ and ${ }^{13} \mathrm{C}\{1 \mathrm{H}\} \mathrm{NMR}$ spectra. Corresponding pairs of resonances were marked with (I) and (II), respectively.

3.2. General Procedure for the Synthesis of 5-Diethoxyphosphoryl-6-methyl-4-phenyl-3,4dihydropyrimidin-2(1H)-one (11a) under Thermal Heating

A mixture of $1.0 \mathrm{mmol}(0.19 \mathrm{~mL})$ diethyl (2-oxopropyl)phosphonate, $1.0 \mathrm{mmol}(0.10 \mathrm{~mL})$ benzaldehyde, $1.5 \mathrm{mmol}(0.09 \mathrm{~g})$ urea and catalyst [ $p$-toluenesulfonic acid $(0.50 \mathrm{mmol}$, $0.10 \mathrm{~g})$ or zinc triflate $(0.10 \mathrm{mmol}, 0.04 \mathrm{~g}$ or $0.15 \mathrm{mmol}, 0.05 \mathrm{~g}$ or $0.20 \mathrm{mmol}, 0.07 \mathrm{~g})$ or scandium triflate $(0.15 \mathrm{mmol}, 0.07 \mathrm{~g})$ or ytterbium triflate $(0.15 \mathrm{mmol}, 0.09 \mathrm{~g})]$ in $2 \mathrm{~mL}$ of acetonitrile, toluene or without any solvent was heated at $82-110{ }^{\circ} \mathrm{C}$ for $2-4 \mathrm{~h}$ in a tube in an oil bath. The volatile components were removed in vacuum, and the residue was analysed by ${ }^{31} \mathrm{P}$ NMR and HPLC-MS.

3.3. General Procedure for the Synthesis of 5-Diethoxyphosphoryl-6-methyl-4-phenyl-3,4dihydropyrimidin-2(1H)-one (11a) in a Microwave Reactor

A mixture of $1.0 \mathrm{mmol}(0.19 \mathrm{~mL})$ diethyl (2-oxopropyl)phosphonate, $1.0 \mathrm{mmol}(0.10 \mathrm{~mL})$, $1.5 \mathrm{mmol}(0.15 \mathrm{~mL})$ or $2.0 \mathrm{mmol}(0.21 \mathrm{~mL})$ benzaldehyde, $1.5 \mathrm{mmol}(0.09 \mathrm{~g}), 2 \mathrm{mmol}(0.12 \mathrm{~g})$ or $2.5 \mathrm{mmol}(0.15 \mathrm{~g})$ urea and $0.15 \mathrm{mmol}(0.05 \mathrm{~g})$ zinc triflate was irradiated in a sealed tube at $100{ }^{\circ} \mathrm{C}$ for 2-4 h in a CEM Microwave reactor (CEM Microwave Technology Ltd., Buckingham, UK) equipped with a pressure controller. The volatile components were removed in vacuum, and the residue was analyzed by ${ }^{31} \mathrm{P}$ NMR and HPLC-MS.

3.4. General Procedure for the Synthesis of 5-Phosphonato-3,4-dihydropyrimidin-2(1H)-one (11, 13-16) Derivatives

A mixture of $1.0 \mathrm{mmol} \beta$-ketophosphonate (diethyl (2-oxopropyl)phosphonate $(0.19 \mathrm{~mL})$ or $0.14 \mathrm{~mL}$ dimethyl (2-oxopropyl)phosphonate), $1.5 \mathrm{mmol}$ aldehyde $(0.15 \mathrm{~mL}$ benzalde- 
hyde, $0.17 \mathrm{~mL}$ 2-chlorobenzaldehyde, $0.17 \mathrm{~mL}$ 3-chlorobenzaldehyde, $0.17 \mathrm{~mL}$ 4-chlorobenzaldehyde, $0.16 \mathrm{~mL}$ 4-fluorobenzaldehde, $0.38 \mathrm{~g}$ 2-fluoro-4-iodobenzaldehyde, $0.18 \mathrm{~mL} 3$-methylbenzaldehyde, $0.18 \mathrm{~g}$ 4-hydroxybenzaldehyde, $0.23 \mathrm{~g}$ 4-nitrobenzaldehyde, $0.29 \mathrm{~g}$ 3,4,5-trimethoxybenzaldehyde, $0.14 \mathrm{~mL}$ butyraldehyde or $0.16 \mathrm{~mL}$ isovaleraldehyde), $2.0 \mathrm{mmol}$ urea derivative (0.12 $\mathrm{g}$ urea or $0.14 \mathrm{~g} \mathrm{~N}$-methylurea) and $0.15 \mathrm{mmol}(0.05 \mathrm{~g})$ zinc triflate was irradiated in a sealed tube at $100{ }^{\circ} \mathrm{C}$ for $2 \mathrm{~h}$ in a CEM Microwave reactor equipped with a pressure controller. The volatile components were removed in vacuum, and the residue was analyzed by ${ }^{31} \mathrm{P}$ NMR and HPLC-MS. The 5-phosphonato3,4-dihydropyrimidin-2(1H)-ones $(\mathbf{4}, \mathbf{6 - 8})$ were obtained by column chromatography using silica gel as the absorbent and dichloromethane:methanol (9:1) as the eluent, or by reversedphase column chromatography using C18-reversed phase silica gel and the composition of eluent A was $0.1 \%(\mathrm{NH} 4)(\mathrm{HCOO})$ in water; eluent B was $0.1 \%(\mathrm{NH} 4)(\mathrm{HCOO})$ and $8 \%$ water in acetonitrile. The following products were thus prepared:

5-Diethoxyphosphoryl-6-methyl-4-phenyl-3,4-dihydropyrimidin-2(1H)-one (11a): Yield: 75\% (0.24 g), white solid; Mp: 168-169 ${ }^{\circ} \mathrm{C}$; Mp [27]: $169-170{ }^{\circ} \mathrm{C} ;{ }^{31} \mathrm{P}\left(\mathrm{DMSO}-\mathrm{d}_{6}\right) \delta 19.8 ;{ }^{31} \mathrm{P}$ $\left(\mathrm{DMSO}_{6}\right) \delta[27] 19.1 ;[\mathrm{M}+\mathrm{H}]^{+}$found $=325.1314,[\mathrm{M}+\mathrm{H}]^{+}$calculated $=325.1317$.

5-Diethoxyphosphoryl-6-methyl-4-(2-chlorophenyl)-3,4-dihydropyrimidin-2(1H)-one (11b): Yield: 66\% (0.24 g), white solid; Mp: $230-231{ }^{\circ} \mathrm{C} ;{ }^{31} \mathrm{P}\left(\mathrm{DMSO}_{6} \mathrm{~d}_{6}\right) \delta 19.0 ;{ }^{13} \mathrm{C}$ NMR $\left(\mathrm{DMSO}-\mathrm{d}_{6}\right) \delta$ $16.1\left(\mathrm{~d},{ }^{3} J_{\mathrm{CP}}=7.0, \mathrm{CH}_{3} \mathrm{CH}_{2} \mathrm{O}^{\mathrm{I}}\right), 16.6\left(\mathrm{~d},{ }^{3} J_{\mathrm{CP}}=6.4, \mathrm{CH}_{3} \mathrm{CH}_{2} \mathrm{O}^{\mathrm{II}}\right), 17.7\left(\mathrm{~d}^{3} J_{\mathrm{CP}}=3.4, \mathrm{CCH}_{3}\right)$, $52.3\left(\mathrm{~d},{ }^{2} J_{\mathrm{CP}}=15.1, \mathrm{CHNH}\right), 60.8\left(\mathrm{~d},{ }^{2} J_{\mathrm{CP}}=5.0, \mathrm{CH}_{3} \mathrm{CH}_{2} \mathrm{O}^{\mathrm{I}}\right), 61.1\left(\mathrm{~d},{ }^{2} J_{\mathrm{CP}}=5.2, \mathrm{CH}_{3} \mathrm{CH}_{2} \mathrm{O}^{\mathrm{II}}\right)$, $93.1\left(\mathrm{~d},{ }^{1} J_{\mathrm{CP}}=207.0, \mathrm{PC}=\mathrm{C}\right), 128.1\left(\mathrm{C}_{6}\right), 129.5\left(\mathrm{C}_{4}\right), 129.7\left(\mathrm{C}_{5}\right), 129.8\left(\mathrm{C}_{3}\right), 132.1\left(\mathrm{C}_{2}\right), 141.6$ $\left(\mathrm{C}_{1}\right), 149.8\left(\mathrm{~d},{ }^{2} J_{\mathrm{CP}}=21.8, \mathrm{PC}=\mathrm{C}\right), 152.4(\mathrm{C}=\mathrm{O}) ;{ }^{1} \mathrm{H}$ NMR $\left(\mathrm{DMSO}-\mathrm{d}_{6}\right) \delta 0.85\left(\mathrm{t}, J_{\mathrm{HH}}=7.0,3 \mathrm{H}\right.$, $\left.\mathrm{CH}_{3} \mathrm{CH}_{2} \mathrm{O}^{\mathrm{I}}\right), 1.18\left(\mathrm{t}, J_{\mathrm{HH}}=7.1,3 \mathrm{H}, \mathrm{CH}_{3} \mathrm{CH}_{2} \mathrm{O}^{\mathrm{II}}\right), 2.20\left(\mathrm{~d}, J_{\mathrm{HP}}=2.0,3 \mathrm{H}, \mathrm{CCH}_{3}\right), 3.36-3.47(\mathrm{~m}$, $\left.1 \mathrm{H}, \mathrm{CH}_{\mathrm{A}}, \mathrm{CH}_{3} \mathrm{CH}_{2} \mathrm{O}^{\mathrm{I}}\right), 3.59-3.76\left(\mathrm{~m}, 1 \mathrm{H}, \mathrm{CH}_{\mathrm{B}}, \mathrm{CH}_{3} \mathrm{CH}_{2} \mathrm{O}^{\mathrm{I}}\right), 3.76-3.92\left(\mathrm{~m}, 2 \mathrm{H}, \mathrm{CH}_{3} \mathrm{CH}_{2} \mathrm{O}^{\mathrm{II}}\right)$, $5.36\left(\mathrm{dd},{ }^{3} J_{\mathrm{HP}}=8.5, J_{\mathrm{HH}}=3.3,1 \mathrm{H}, \mathrm{CHNH}\right), 7.20-7.49(\mathrm{~m}, 4 \mathrm{H}, \mathrm{ArH}), 7.64($ br s, $1 \mathrm{H}, \mathrm{NH})$, $9.18\left(\mathrm{~d}, J_{\mathrm{HH}}=4.6,1 \mathrm{H}, \mathrm{NH}\right) ;[\mathrm{M}+\mathrm{H}]^{+}$found $=359.0924,[\mathrm{M}+\mathrm{H}]^{+}$calculated $=359.0927$.

5-Diethoxyphosphoryl-6-methyl-4-(3-chlorophenyl)-3,4-dihydropyrimidin-2(1H)-one (11c): Yield: $75 \%$ (0.27 g), light yellow solid; Mp: $170-171{ }^{\circ} \mathrm{C}, \mathrm{Mp}[28]: 171-172{ }^{\circ} \mathrm{C} ;{ }^{31} \mathrm{P}\left(\mathrm{DMSOd}_{6}\right) \delta 19.5$; ${ }^{13} \mathrm{C} \mathrm{NMR}\left(\mathrm{DMSO}_{-} \mathrm{d}_{6}\right) \delta 16.3\left(\mathrm{~d},{ }^{3} \mathrm{~J}_{\mathrm{CP}}=6.7, \mathrm{CH}_{3} \mathrm{CH}_{2} \mathrm{O}^{\mathrm{I}}\right), 16.5\left(\mathrm{~d},{ }^{3} \mathrm{~J}_{\mathrm{CP}}=6.4, \mathrm{CH}_{3} \mathrm{CH}_{2} \mathrm{O}^{\mathrm{II}}\right)$, $17.9\left(\mathrm{~d},{ }^{3} J_{\mathrm{CP}}=3.4, \mathrm{CCH}_{3}\right), 55.1\left(\mathrm{~d},{ }^{2} J_{\mathrm{CP}}=15.2, \mathrm{CHNH}\right), 61.1\left(\mathrm{~d},{ }^{2} J_{\mathrm{CP}}=5.1, \mathrm{CH}_{3} \mathrm{CH}_{2} \mathrm{O}^{\mathrm{I}}\right)$, $61.2\left(\mathrm{~d},{ }^{2} J_{\mathrm{CP}}=5.2, \mathrm{CH}_{3} \mathrm{CH}_{2} \mathrm{O}^{\mathrm{II}}\right), 93.8\left(\mathrm{~d},{ }^{1} J_{\mathrm{CP}}=206.3, \mathrm{PC}=\mathrm{C}\right), 125.6\left(\mathrm{C}_{6}\right), 126.9\left(\mathrm{C}_{2}\right), 127.9$ $\left(\mathrm{C}_{4}\right), 130.9\left(\mathrm{C}_{5}\right), 133.4\left(\mathrm{C}_{3}\right), 147.4\left(\mathrm{C}_{1}\right), 149.3\left(\mathrm{~d},{ }^{2} J_{\mathrm{CP}}=20.7, \mathrm{PC}=\mathrm{C}\right), 152.9(\mathrm{C}=\mathrm{O}) ;{ }^{1} \mathrm{H}$ NMR $\left(\mathrm{DMSO}_{-} \mathrm{d}_{6}\right) \delta 1.00\left(\mathrm{t}, \mathrm{J}_{\mathrm{HH}}=6.3,3 \mathrm{H}, \mathrm{CH}_{3} \mathrm{CH}_{2} \mathrm{O}^{\mathrm{I}}\right), 1.14\left(\mathrm{t}, \mathrm{J}_{\mathrm{HH}}=7.0,3 \mathrm{H}, \mathrm{CH}_{3} \mathrm{CH}_{2} \mathrm{O}^{\mathrm{II}}\right), 2.11$ $\left(\mathrm{d}, J_{\mathrm{HP}}=2.2,3 \mathrm{H}, \mathrm{CCH}_{3}\right), 3.55-3.68\left(\mathrm{~m}, 1 \mathrm{H}, \mathrm{CH}_{\mathrm{A}}, \mathrm{CH}_{3} \mathrm{CH}_{2} \mathrm{O}^{\mathrm{I}}\right), 3.72-3.87\left(\mathrm{~m}, 3 \mathrm{H}, \mathrm{CH}_{\mathrm{B}}\right.$, $\left.\mathrm{CH}_{3} \mathrm{CH}_{2} \mathrm{O}^{\mathrm{I}}, \mathrm{CH}_{3} \mathrm{CH}_{2} \mathrm{O}^{\mathrm{II}}\right), 4.88\left(\mathrm{dd},{ }^{3} \mathrm{~J}_{\mathrm{HP}}=8.8, J_{\mathrm{HH}}=3.6,1 \mathrm{H}, \mathrm{CHNH}\right), 7.15-7.45(\mathrm{~m}, 4 \mathrm{H}$, $\mathrm{ArH}), 7.70($ br s, $1 \mathrm{H}, \mathrm{NH}), 9.19\left(\mathrm{~d}, \mathrm{~J}_{\mathrm{HH}}=4.6,1 \mathrm{H}, \mathrm{NH}\right) ;{ }^{1} \mathrm{H} \mathrm{NMR}\left(\mathrm{CDCl}_{3}+\mathrm{DMSO}^{\mathrm{d}} \mathrm{d}_{6}\right) \delta[28]$ $1.10\left(\mathrm{t}, \mathrm{J}_{\mathrm{HH}}=6.9,3 \mathrm{H}, \mathrm{CH}_{3} \mathrm{CH}_{2} \mathrm{O}^{\mathrm{I}}\right), 1.22\left(\mathrm{t}, \mathrm{J}_{\mathrm{HH}}=6.9,3 \mathrm{H}, \mathrm{CH}_{3} \mathrm{CH}_{2} \mathrm{O}^{\mathrm{II}}\right), 2.17\left(\mathrm{~s}, 3 \mathrm{H}, \mathrm{CCH}_{3}\right)$, 3.80-3.91 (m, 4H, $\left.\mathrm{CH}_{3} \mathrm{CH}_{2} \mathrm{O}\right), 4.96-4.99(\mathrm{~m}, 1 \mathrm{H}, \mathrm{CHNH}), 7.24-7.34$ (m, 3H, ArH), 7.63 (s, $1 \mathrm{H}, \mathrm{ArH}), 8.01(\mathrm{~s}, 1 \mathrm{H}, \mathrm{NH}), 9.12(\mathrm{~s}, 1 \mathrm{H}, \mathrm{NH}) ;[\mathrm{M}+\mathrm{H}]^{+}$found $=359.0916,[\mathrm{M}+\mathrm{H}]^{+}$calculated $=$ 359.0927.

5-Diethoxyphosphoryl-6-methyl-4-(4-chlorophenyl)-3,4-dihydropyrimidin-2(1H)-one (11d): Yield: 69\% (0.25 g), white solid; Mp: $120-121{ }^{\circ} \mathrm{C}$; Mp [27]: $120-122{ }^{\circ} \mathrm{C} ;{ }^{31} \mathrm{P}\left(\mathrm{DMSO}-\mathrm{d}_{6}\right) \delta 19.6 ;{ }^{31} \mathrm{P}$ $\left(\mathrm{DMSO}-\mathrm{d}_{6}\right) \delta[27] 19.8 ;[\mathrm{M}+\mathrm{H}]^{+}$found $=359.0923,[\mathrm{M}+\mathrm{H}]^{+}$calculated $=359.0927$.

5-Diethoxyphosphoryl-6-methyl-4-(4-fluorophenyl)-3,4-dihydropyrimidin-2(1H)-one (11e): Yield: 79\% (0.27 g), white solid; Mp: $109-110{ }^{\circ} \mathrm{C} ; \mathrm{Mp}$ [28]: $110-112{ }^{\circ} \mathrm{C} ;{ }^{31} \mathrm{P}$ NMR (DMSO-d 6$) \delta 19.7$; ${ }^{13} \mathrm{C} \mathrm{NMR}\left(\mathrm{DMSO}-\mathrm{d}_{6}\right) \delta 16.3\left(\mathrm{~d},{ }^{3} J_{\mathrm{CP}}=6.7, \mathrm{CH}_{3} \mathrm{CH}_{2} \mathrm{O}^{\mathrm{I}}\right), 16.5\left(\mathrm{~d},{ }^{3} \mathrm{~J}_{\mathrm{CP}}=6.2, \mathrm{CH}_{3} \mathrm{CH}_{2} \mathrm{O}^{\mathrm{II}}\right)$, $17.8\left(\mathrm{~d},{ }^{3} J_{\mathrm{CP}}=3.4, \mathrm{CCH}_{3}\right), 54.9\left(\mathrm{~d},{ }^{2} J_{\mathrm{CP}}=15.0, \mathrm{CHNH}\right), 61.0\left(\mathrm{~d},{ }^{2} \mathrm{~J}_{\mathrm{CP}}=5.0, \mathrm{CH}_{3} \mathrm{CH}_{2} \mathrm{O}^{\mathrm{I}}\right), 61.1$ $\left(\mathrm{d},{ }^{2} J_{\mathrm{CP}}=5.1, \mathrm{CH}_{3} \mathrm{CH}_{2} \mathrm{O}^{\mathrm{II}}\right), 94.2\left(\mathrm{~d},{ }^{1} J_{\mathrm{CP}}=205.5, \mathrm{PC}=\mathrm{C}\right), 115.5\left(\mathrm{~d},{ }^{2} J_{\mathrm{CF}}=21.4, \mathrm{C}_{3}\right), 129.0(\mathrm{~d}$, $\left.{ }^{3} J_{\mathrm{CF}}=8.3, \mathrm{C}_{2}\right), 141.3\left(\mathrm{~d}, J_{\mathrm{CF}}=2.5, \mathrm{C}_{1}\right), 148.8\left(\mathrm{~d},{ }^{2} J_{\mathrm{CP}}=20.7, \mathrm{PC}=\mathrm{C}\right), 152.9(\mathrm{C}=\mathrm{O}), 161.9(\mathrm{~d}$, $\left.{ }^{1} J_{\mathrm{CF}}=243.0, \mathrm{C}_{4}\right) ;{ }^{1} \mathrm{H}$ NMR $\left(\mathrm{DMSO}-\mathrm{d}_{6}\right) \delta 0.98\left(\mathrm{t}, \mathrm{J}_{\mathrm{HH}}=7.0,3 \mathrm{H}, \mathrm{CH}_{3} \mathrm{CH}_{2} \mathrm{O}^{\mathrm{I}}\right), 1.13\left(\mathrm{t}, J_{\mathrm{HH}}=7.0\right.$, $\left.3 \mathrm{H}, \mathrm{CH}_{3} \mathrm{CH}_{2} \mathrm{O}^{\mathrm{II}}\right), 2.10\left(\mathrm{~d}, \mathrm{~J}_{\mathrm{HP}}=2.5,3 \mathrm{H}, \mathrm{CCH}_{3}\right), 3.49-3.65\left(\mathrm{~m}, 1 \mathrm{H}, \mathrm{CH}_{\mathrm{A}}, \mathrm{CH}_{3} \mathrm{CH}_{2} \mathrm{O}^{\mathrm{I}}\right), 3.68-$ $3.85\left(\mathrm{~m}, 3 \mathrm{H}, \mathrm{CH}_{\mathrm{B}}, \mathrm{CH}_{3} \mathrm{CH}_{2} \mathrm{O}^{\mathrm{I}}, \mathrm{CH}_{3} \mathrm{CH}_{2} \mathrm{O}^{\mathrm{II}}\right), 4.86\left(\mathrm{dd},{ }^{3} J_{\mathrm{HP}}=8.6, J_{\mathrm{HH}}=3.5,1 \mathrm{H}, \mathrm{CHNH}\right)$, 
7.10-7.22 (m, 2H, ArH), 7.24-7.37 (m, 2H, ArH), 7.65 (br s, 1H, NH), $9.15\left(\mathrm{~d}, J_{\mathrm{HH}}=4.7\right.$, $1 \mathrm{H}, \mathrm{NH}) ;{ }^{1} \mathrm{H}$ NMR $\left(\mathrm{CDCl}_{3}+\mathrm{DMSO}_{6}\right) \delta[28] 1.04\left(\mathrm{t}, \mathrm{J}_{\mathrm{HH}}=7.0,3 \mathrm{H}, \mathrm{CH}_{3} \mathrm{CH}_{2} \mathrm{O}^{\mathrm{I}}\right), 1.19$ $\left(\mathrm{t}, \mathrm{J}_{\mathrm{HH}}=7.0,3 \mathrm{H}, \mathrm{CH}_{3} \mathrm{CH}_{2} \mathrm{O}^{\mathrm{II}}\right), 2.13\left(\mathrm{~s}, 3 \mathrm{H}, \mathrm{CCH}_{3}\right), 3.70-3.85\left(\mathrm{~m}, 4 \mathrm{H}, \mathrm{CH}_{3} \mathrm{CH}_{2} \mathrm{O}\right), 4.92$ $\left(\mathrm{dd},{ }^{3} J_{\mathrm{HP}}=8.5, J_{\mathrm{HH}}=3.2,1 \mathrm{H}, \mathrm{CHNH}\right), 7.04-7.34(\mathrm{~m}, 4 \mathrm{H}, \mathrm{ArH}), 7.60(\mathrm{~s}, 1 \mathrm{H}, \mathrm{NH}), 9.12(\mathrm{~d}$, $\left.J_{\mathrm{HH}}=3.6,1 \mathrm{H}, \mathrm{NH}\right) ;[\mathrm{M}+\mathrm{H}]^{+}$found $=343.1221,[\mathrm{M}+\mathrm{H}]^{+}$calculated $=343.1222$.

5-Diethoxyphosphoryl-6-methyl-4-(2-fluoro-4-iodophenyl)-3,4-dihydropyrimidin-2(1H)-one (11f): Yield: $61 \%$ (0.29 g), white solid; Mp: $103-104{ }^{\circ} \mathrm{C} ;{ }^{31} \mathrm{P}\left(\mathrm{DMSO}_{6}\right) \delta 25.9 ;{ }^{13} \mathrm{C}$ NMR (DMSO$\left.\mathrm{d}_{6}\right) \delta 20.1\left(\mathrm{~d},{ }^{3} J_{\mathrm{CP}}=7.0, \mathrm{CH}_{3} \mathrm{CH}_{2} \mathrm{O}^{\mathrm{I}}\right), 20.5\left(\mathrm{~d},{ }^{3} J_{\mathrm{CP}}=6.2, \mathrm{CH}_{3} \mathrm{CH}_{2} \mathrm{O}^{\mathrm{II}}\right), 21.8\left(\mathrm{~d},{ }^{3} J_{\mathrm{CP}}=3.5\right.$, $\left.\mathrm{CCH}_{3}\right), 53.3\left(\mathrm{dd},{ }^{2} J_{\mathrm{CP}}=24.4,{ }^{3} J_{\mathrm{CF}}=2.6, \mathrm{CHNH}\right), 64.9\left(\mathrm{~d},{ }^{2} J_{\mathrm{CP}}=5.1, \mathrm{CH}_{3} \mathrm{CH}_{2} \mathrm{O}^{\mathrm{I}}\right), 65.1$ $\left(\mathrm{d},{ }^{2} J_{\mathrm{CP}}=5.2, \mathrm{CH}_{3} \mathrm{CH}_{2} \mathrm{O}^{\mathrm{II}}\right), 96.0\left(\mathrm{~d},{ }^{1} J_{\mathrm{CP}}=207.0, \mathrm{PC}=\mathrm{C}\right), 97.8\left(\mathrm{~d},{ }^{3} J_{\mathrm{CF}}=8.7, \mathrm{C}_{6}\right), 128.7(\mathrm{~d}$, $\left.{ }^{2} J_{\mathrm{CF}}=24.8, \mathrm{C}_{3}\right), 135.3\left(\mathrm{~d},{ }^{3} J_{\mathrm{CF}}=4.0, \mathrm{C}_{5}\right), 135.7\left(\mathrm{~d},{ }^{2} J_{\mathrm{CF}}=13.8, \mathrm{C}_{1}\right), 138.0\left(\mathrm{~d},{ }^{3} J_{\mathrm{CF}}=3.4, \mathrm{C}_{4}\right)$, $153.6\left(\mathrm{~d},{ }^{2} J_{\mathrm{CP}}=21.4, \mathrm{PC}=\mathrm{C}\right), 156.3(\mathrm{C}=\mathrm{O}), 163.5\left(\mathrm{~d},{ }^{1} J_{\mathrm{CF}}=252.0, \mathrm{C}_{2}\right) ;{ }^{1} \mathrm{H}$ NMR $\left(\mathrm{DMSO}-\mathrm{d}_{6}\right) \delta$ $0.93\left(\mathrm{t}, \mathrm{J}_{\mathrm{HH}}=7.0,3 \mathrm{H}, \mathrm{CH}_{3} \mathrm{CH}_{2} \mathrm{O}^{\mathrm{I}}\right), 1.14\left(\mathrm{t}, \mathrm{J}_{\mathrm{HH}}=7.1,3 \mathrm{H}, \mathrm{CH}_{3} \mathrm{CH}_{2} \mathrm{O}^{\mathrm{II}}\right), 2.13\left(\mathrm{~d}, J_{\mathrm{HP}}=2.4\right.$, $\left.3 \mathrm{H}, \mathrm{CCH}_{3}\right), 3.43-3.58\left(\mathrm{~m}, 1 \mathrm{H}, \mathrm{CH}_{\mathrm{A}}, \mathrm{CH}_{3} \mathrm{CH}_{2} \mathrm{O}^{\mathrm{I}}\right), 3.64-3.88,\left(\mathrm{~m}, 3 \mathrm{H}, \mathrm{CH}_{\mathrm{B}}, \mathrm{CH}_{3} \mathrm{CH}_{2} \mathrm{O}^{\mathrm{I}}\right.$, $\left.\mathrm{CH}_{3} \mathrm{CH}_{2} \mathrm{O}^{\mathrm{II}}\right), 5.14\left(\mathrm{dd},{ }^{3} J_{\mathrm{HP}}=8.0, J_{\mathrm{HH}}=3.2,1 \mathrm{H}, \mathrm{CHNH}\right), 7.07\left(\mathrm{t}, J_{\mathrm{HH}}=8.0,1 \mathrm{H}, \mathrm{ArH}\right)$, 7.47-7.78 (m, 3H, ArH, NH), $9.16\left(\mathrm{~d}, J_{\mathrm{HH}}=3.0,1 \mathrm{H}, \mathrm{NH}\right) ;[\mathrm{M}+\mathrm{H}]^{+}$found $=469.0184,[\mathrm{M}+$ $\mathrm{H}]^{+}$calculated $=469.0189$.

5-Diethoxyphosphoryl-6-methyl-4-(3-methylphenyl)-3,4-dihydropyrimidin-2(1H)-one (11g): Yield: $72 \%$ (0.24 g), white solid; Mp: $192-193{ }^{\circ} \mathrm{C} ;{ }^{31} \mathrm{P}\left(\mathrm{DMSO}_{6} \mathrm{~d}_{6}\right) \delta 19.9 ;{ }^{13} \mathrm{C}$ NMR $\left(\mathrm{DMSO}-\mathrm{d}_{6}\right) \delta$ $16.2\left(\mathrm{~d},{ }^{3} J_{\mathrm{CP}}=6.8, \mathrm{CH}_{3} \mathrm{CH}_{2} \mathrm{O}^{\mathrm{I}}\right), 16.5\left(\mathrm{~d},{ }^{3} J_{\mathrm{CP}}=6.3, \mathrm{CH}_{3} \mathrm{CH}_{2} \mathrm{O}^{\mathrm{II}}\right), 17.9\left(\mathrm{~d},{ }^{3} J_{\mathrm{CP}}=3.5, \mathrm{CCH}_{3}\right)$, $21.6\left(\mathrm{~s}, \mathrm{C}_{3} \mathrm{CH}_{3}\right), 55.5\left(\mathrm{~d},{ }^{2} J_{\mathrm{CP}}=15.1, \mathrm{CHNH}\right), 60.9\left(\mathrm{~d},{ }^{2} J_{\mathrm{CP}}=4.9, \mathrm{CH}_{3} \mathrm{CH}_{2} \mathrm{O}^{\mathrm{I}}\right), 61.0(\mathrm{~d}$, $\left.{ }^{2} J_{\mathrm{CP}}=5.1, \mathrm{CH}_{3} \mathrm{CH}_{2} \mathrm{O}^{\mathrm{II}}\right), 94.2\left(\mathrm{~d},{ }^{1} J_{\mathrm{CP}}=205.3, \mathrm{PC}=\mathrm{C}\right), 124.1\left(\mathrm{C}_{6}\right), 127.6\left(\mathrm{C}_{2}\right), 128.4\left(\mathrm{C}_{4}\right), 128.7$ $\left(\mathrm{C}_{5}\right), 137.7\left(\mathrm{C}_{3}\right), 144.9\left(\mathrm{C}_{1}\right), 148.7\left(\mathrm{~d},{ }^{2} \mathrm{~J}_{\mathrm{CP}}=20.7, \mathrm{PC}=\mathrm{C}\right), 153.0(\mathrm{C}=\mathrm{O}) ;{ }^{1} \mathrm{H}$ NMR $\left(\mathrm{DMSO}-\mathrm{d}_{6}\right)$ $\delta 0.98\left(\mathrm{t}, J_{\mathrm{HH}}=7.1,3 \mathrm{H}, \mathrm{CH}_{3} \mathrm{CH}_{2} \mathrm{O}^{\mathrm{I}}\right), 1.14\left(\mathrm{t}, \mathrm{J}_{\mathrm{HH}}=7.1,3 \mathrm{H}, \mathrm{CH}_{3} \mathrm{CH}_{2} \mathrm{O}^{\mathrm{II}}\right), 2.11\left(\mathrm{~d}, J_{\mathrm{HP}}=2.3\right.$, $\left.3 \mathrm{H}, \mathrm{CCH}_{3}\right), 2.28\left(\mathrm{~s}, 3 \mathrm{H}, \mathrm{C}_{3} \mathrm{CH}_{3}\right), 3.51-3.60\left(\mathrm{~m}, 1 \mathrm{H}, \mathrm{CH}_{\mathrm{A}}, \mathrm{CH}_{3} \mathrm{CH}_{2} \mathrm{O}^{\mathrm{I}}\right), 3.72-3.82(\mathrm{~m}, 3 \mathrm{H}$, $\left.\mathrm{CH}_{\mathrm{B}}, \mathrm{CH}_{3} \mathrm{CH}_{2} \mathrm{O}^{\mathrm{I}}, \mathrm{CH}_{3} \mathrm{CH}_{2} \mathrm{O}^{\mathrm{II}}\right), 4.82\left(\mathrm{dd},{ }^{3} \mathrm{~J}_{\mathrm{HP}}=8.7, J_{\mathrm{HH}}=3.4,1 \mathrm{H}, \mathrm{CHNH}\right), 7.07-7.13(\mathrm{~m}$, $3 \mathrm{H}, \mathrm{ArH}), 7.19-7.25$ (m, 1H, ArH), 7.60 (br s, 1H, NH), 9.11 (d, JHH $=3.1,1 \mathrm{H}, \mathrm{NH})$; $[\mathrm{M}+$ $\mathrm{H}]^{+}$found $=339.1473,[\mathrm{M}+\mathrm{H}]^{+}$calculated $=339.1473$.

5-Diethoxyphosphoryl-6-methyl-4-(4-hydroxyphenyl)-3,4-dihydropyrimidin-2(1H)-one (11h): Yield: $62 \%$ (0.21 g), yellow solid; Mp: $186-188{ }^{\circ} \mathrm{C} ;{ }^{31} \mathrm{P}\left(\mathrm{DMSO}_{\mathrm{d}}\right) \delta 20.1 ;{ }^{13} \mathrm{C} \mathrm{NMR}\left(\mathrm{DMSO}-\mathrm{d}_{6}\right)$ $\delta 16.3\left(\mathrm{~d},{ }^{3} J_{\mathrm{CP}}=7.0, \mathrm{CH}_{3} \mathrm{CH}_{2} \mathrm{O}^{\mathrm{I}}\right), 16.6\left(\mathrm{~d},{ }^{3} \mathrm{~J}_{\mathrm{CP}}=6.3, \mathrm{CH}_{3} \mathrm{CH}_{2} \mathrm{O}^{\mathrm{II}}\right), 17.8\left(\mathrm{~d},{ }^{3} J_{\mathrm{CP}}=3.7\right.$, $\left.\mathrm{CCH}_{3}\right), 55.0\left(\mathrm{~d},{ }^{2} J_{\mathrm{CP}}=14.8, \mathrm{CHNH}\right), 60.9\left(\mathrm{~d},{ }^{2} J_{\mathrm{CP}}=4.5, \mathrm{CH}_{3} \mathrm{CH}_{2} \mathrm{O}^{\mathrm{I}}\right), 61.0\left(\mathrm{~d},{ }^{2} J_{\mathrm{CP}}=4.7\right.$, $\left.\mathrm{CH}_{3} \mathrm{CH}_{2} \mathrm{O}^{\mathrm{II}}\right), 94.7\left(\mathrm{~d},{ }^{1} J_{\mathrm{CP}}=204.6, \mathrm{PC}=\mathrm{C}\right), 115.4\left(\mathrm{C}_{3}\right), 127.7\left(\mathrm{C}_{2}\right), 135.6\left(\mathrm{C}_{1}\right), 148.2(\mathrm{~d}$, $\left.{ }^{2} J_{\mathrm{CP}}=21.0, \mathrm{PC}=\mathrm{C}\right), 153.1(\mathrm{C}=\mathrm{O}), 157.1\left(\mathrm{C}_{4}\right) ;{ }^{1} \mathrm{H}$ NMR $\left(\mathrm{DMSO}-\mathrm{d}_{6}\right) \delta 0.92\left(\mathrm{t}, J_{\mathrm{HH}}=7.0,3 \mathrm{H}\right.$, $\left.\mathrm{CH}_{3} \mathrm{CH}_{2} \mathrm{O}^{\mathrm{I}}\right), 1.07\left(\mathrm{t}, \mathrm{J}_{\mathrm{HH}}=7.0,3 \mathrm{H}, \mathrm{CH}_{3} \mathrm{CH}_{2} \mathrm{O}^{\mathrm{II}}\right), 2.03\left(\mathrm{~d}, \mathrm{~J}_{\mathrm{HP}}=2.1,3 \mathrm{H}, \mathrm{CCH}_{3}\right), 3.41-3.52$ $\left(\mathrm{m}, 1 \mathrm{H}, \mathrm{CH}_{\mathrm{A}}, \mathrm{CH}_{3} \mathrm{CH}_{2} \mathrm{O}^{\mathrm{I}}\right), 3.61-3.77\left(\mathrm{~m}, 3 \mathrm{H}, \mathrm{CH}_{\mathrm{B}}, \mathrm{CH}_{3} \mathrm{CH}_{2} \mathrm{O}^{\mathrm{I}}, \mathrm{CH}_{3} \mathrm{CH}_{2} \mathrm{O}^{\mathrm{II}}\right), 4.67$ (dd, $\left.{ }^{3} J_{\mathrm{HP}}=9.1, J_{\mathrm{HH}}=3.3,1 \mathrm{H}, \mathrm{CHNH}\right), 6.53-6.72(\mathrm{~m}, 2 \mathrm{H}, \mathrm{ArH}), 6.93-7.09$ (m, 2H, ArH), 7.45 (br s, $1 \mathrm{H}, \mathrm{NH}), 8.98($ br s, $1 \mathrm{H}, \mathrm{NH}), 9.28(\mathrm{~s}, 1 \mathrm{H}, \mathrm{OH}) ;[\mathrm{M}+\mathrm{H}]^{+}$found $=341.1262,[\mathrm{M}+\mathrm{H}]^{+}$calculated $=341.1266$.

5-Diethoxyphosphoryl-6-methyl-4-(4-nitrophenyl)-3,4-dihydropyrimidin-2(1H)-one (11i): Yield: 73\% (0.27 g), yellow solid; Mp: $218-219{ }^{\circ} \mathrm{C}$; Mp [27]: $218-220{ }^{\circ} \mathrm{C} ;{ }^{31} \mathrm{P}\left(\mathrm{DMSO}_{6}\right) \delta 19.1{ }^{31} \mathrm{P}$ $\left(\mathrm{DMSO}-\mathrm{d}_{6}\right) \delta[27] 19.2 ;[\mathrm{M}+\mathrm{H}]^{+}$found $=370.1166,[\mathrm{M}+\mathrm{H}]^{+}$calculated $=370.1167$.

5-Diethoxyphosphoryl-6-methyl-4-(3,4,5-trimethoxyphenyl)-3,4-dihydropyrimidin-2(1H)-one (11j): Yield: $60 \%$ (0.25 g), white solid; Mp: $175-177{ }^{\circ} \mathrm{C} ;{ }^{31} \mathrm{P}\left(\mathrm{DMSO}-\mathrm{d}_{6}\right) \delta 20.0 ;{ }^{13} \mathrm{C}$ NMR (DMSO$\left.\mathrm{d}_{6}\right) \delta 16.3\left(\mathrm{~d},{ }^{3} J_{\mathrm{CP}}=6.6, \mathrm{CH}_{3} \mathrm{CH}_{2} \mathrm{O}^{\mathrm{I}}\right), 16.6\left(\mathrm{~d},{ }^{3} J_{\mathrm{CP}}=6.3, \mathrm{CH}_{3} \mathrm{CH}_{2} \mathrm{O}^{\mathrm{II}}\right), 17.9\left(\mathrm{~d},{ }^{3} J_{\mathrm{CP}}=3.5\right.$, $\left.\mathrm{CCH}_{3}\right), 55.3\left(\mathrm{~d},{ }^{2} J_{\mathrm{CP}}=15.3, \mathrm{CHNH}\right), 56.3\left(\mathrm{C}_{3} \mathrm{OCH}_{3}\right), 60.5\left(\mathrm{C}_{4} \mathrm{OCH}_{3}\right), 61.10\left(\mathrm{~d},{ }^{2} J_{\mathrm{CP}}=5.2\right.$, $\left.\mathrm{CH}_{3} \mathrm{CH}_{2} \mathrm{O}^{\mathrm{I}}\right), 61.13\left(\mathrm{~d},{ }^{2} J_{\mathrm{CP}}=5.6, \mathrm{CH}_{3} \mathrm{CH}_{2} \mathrm{O}^{\mathrm{II}}\right), 94.0\left(\mathrm{~d},{ }^{1} J_{\mathrm{CP}}=206.7, \mathrm{PC}=\mathrm{C}\right), 104.4\left(\mathrm{C}_{2}\right), 137.4$ $\left(\mathrm{C}_{3}\right), 140.4\left(\mathrm{C}_{4}\right), 149.0\left(\mathrm{~d},{ }^{2} J_{\mathrm{CP}}=20.7, \mathrm{PC}=\mathrm{C}\right), 152.7(\mathrm{C}=\mathrm{O}), 153.2\left(\mathrm{C}_{1}\right) ;{ }^{1} \mathrm{H}$ NMR $\left(\mathrm{DMSO}-\mathrm{d}_{6}\right)$ $\delta 1.03\left(\mathrm{t}, J_{\mathrm{HH}}=7.0,3 \mathrm{H}, \mathrm{CH}_{3} \mathrm{CH}_{2} \mathrm{O}^{\mathrm{I}}\right), 1.16\left(\mathrm{t}, \mathrm{J}_{\mathrm{HH}}=7.1,3 \mathrm{H}, \mathrm{CH}_{3} \mathrm{CH}_{2} \mathrm{O}^{\mathrm{II}}\right), 2.11\left(\mathrm{~d}, J_{\mathrm{HP}}=2.5\right.$, $\left.3 \mathrm{H}, \mathrm{CCH}_{3}\right), 3.57-3.90$ [3.63 $\left(\mathrm{s}, \mathrm{C}_{4} \mathrm{OCH}_{3}\right), 3.73\left(\mathrm{~s}, \mathrm{C}_{3} \mathrm{OCH}_{3}\right)$ overlapped by the multiplet of $\mathrm{CH}_{3} \mathrm{CH}_{2} \mathrm{O}$ total int. $\left.13 \mathrm{H}\right], 4.83\left(\mathrm{dd},{ }^{3} J_{\mathrm{HP}}=8.9, J_{\mathrm{HH}}=3.5,1 \mathrm{H}, \mathrm{CHNH}\right), 6.61(\mathrm{~s}, 2 \mathrm{H}, \mathrm{ArH})$, 
$7.62(\mathrm{br} \mathrm{s}, 1 \mathrm{H}, \mathrm{NH}), 9.11\left(\mathrm{~d}, J_{\mathrm{HH}}=3.0,1 \mathrm{H}, \mathrm{NH}\right) ;[\mathrm{M}+\mathrm{H}]^{+}$found $=415.1631,[\mathrm{M}+\mathrm{H}]^{+}$calculated $=415.1634$.

5-Dimethoxyphosphoryl-6-methyl-4-phenyl-3,4-dihydropyrimidin-2(1H)-one (13a): Yield: 80\% (0.24 g), white solid; Mp: 197-199 ${ }^{\circ} \mathrm{C}$; Mp [27]: $198-200{ }^{\circ} \mathrm{C} ;{ }^{31} \mathrm{P}\left(\mathrm{DMSO}-\mathrm{d}_{6}\right) \delta 23.0 ;{ }^{31} \mathrm{P}$ $\left(\mathrm{DMSO}-\mathrm{d}_{6}\right) \delta[27] 22.8 ;[\mathrm{M}+\mathrm{H}]^{+}$found $=297.1001,[\mathrm{M}+\mathrm{H}]^{+}$calculated $=297.1004$.

5-Dimethoxyphosphoryl-6-methyl-4-(2-chlorophenyl)-3,4-dihydropyrimidin-2(1H)-one (13b) Yield: $62 \%$ (0.20 g), white solid; Mp: $230-231{ }^{\circ} \mathrm{C} ;{ }^{31} \mathrm{P}\left(\mathrm{DMSO}_{6} \mathrm{~d}_{6}\right) \delta 22.1 ;{ }^{13} \mathrm{C}$ NMR $\left(\mathrm{DMSO}-\mathrm{d}_{6}\right) \delta$ $17.8\left(\mathrm{~d},{ }^{3} J_{\mathrm{CP}}=3.4, \mathrm{CCH}_{3}\right), 51.7\left(\mathrm{~d},{ }^{2} J_{\mathrm{CP}}=5.1, \mathrm{CH}_{3} \mathrm{O}^{\mathrm{I}}\right), 52.0\left(\mathrm{~d},{ }^{2} J_{\mathrm{CP}}=5.4, \mathrm{CH}_{3} \mathrm{O}^{\mathrm{II}}\right), 52.3(\mathrm{~d}$, $\left.{ }^{2} J_{\mathrm{CP}}=15.0, \mathrm{CHNH}\right), 91.9\left(\mathrm{~d},{ }^{1} J_{\mathrm{CP}}=207.4, \mathrm{PC}=\mathrm{C}\right), 128.1\left(\mathrm{C}_{6}\right), 129.5\left(\mathrm{C}_{4}\right), 129.7\left(\mathrm{C}_{5}\right), 129.9$ $\left(\mathrm{C}_{3}\right), 132.0\left(\mathrm{C}_{2}\right), 141.5\left(\mathrm{C}_{1}\right), 150.4\left(\mathrm{~d},{ }^{2} J_{\mathrm{CP}}=21.7, \mathrm{PC}=\mathrm{C}\right), 152.3(\mathrm{C}=\mathrm{O}) ;{ }^{1} \mathrm{H}$ NMR $\left(\mathrm{DMSO}-\mathrm{d}_{6}\right)$ $\delta 2.18\left(\mathrm{~s}, 3 \mathrm{H}, \mathrm{CCH}_{3}\right), 3.18\left(\mathrm{~d},{ }^{3} J_{\mathrm{HP}}=11.1,3 \mathrm{H}, \mathrm{CH}_{3} \mathrm{O}^{\mathrm{I}}\right), 3.48\left(\mathrm{~d},{ }^{3} \mathrm{~J}_{\mathrm{HP}}=11.2,3 \mathrm{H}, \mathrm{CH}_{3} \mathrm{O}^{\mathrm{II}}\right)$, $5.34\left(\mathrm{dd},{ }^{3} J_{\mathrm{HP}}=8.4, J_{\mathrm{HH}}=3.4,1 \mathrm{H}, \mathrm{CHNH}\right), 7.21-7.50(\mathrm{~m}, 4 \mathrm{H}, \mathrm{ArH}), 7.68($ br s, $1 \mathrm{H}, \mathrm{NH})$, $9.24\left(\mathrm{~d}, J_{\mathrm{HH}}=6.1,1 \mathrm{H}, \mathrm{NH}\right) ;[\mathrm{M}+\mathrm{H}]^{+}$found $=331.0612,[\mathrm{M}+\mathrm{H}]^{+}$calculated $=331.0614$.

5-Dimethoxyphosphoryl-6-methyl-4-(3-chlorophenyl)-3,4-dihydropyrimidin-2(1H)-one (13c): Yield: $75 \%$ (0.25 g), white solid; Mp: $126-127{ }^{\circ} \mathrm{C} ;{ }^{31} \mathrm{P}\left(\mathrm{DMSO}_{6} \mathrm{~d}_{6}\right) \delta 22.6 ;{ }^{13} \mathrm{C}$ NMR $\left(\mathrm{DMSO}-\mathrm{d}_{6}\right) \delta$ $17.9\left(\mathrm{~d},{ }^{3} J_{\mathrm{CP}}=3.6, \mathrm{CCH}_{3}\right), 51.9\left(\mathrm{~d},{ }^{2} J_{\mathrm{CP}}=5.0, \mathrm{CH}_{3} \mathrm{O}^{\mathrm{I}}\right), 52.1\left(\mathrm{~d},{ }^{2} J_{\mathrm{CP}}=5.3, \mathrm{CH}_{3} \mathrm{O}^{\mathrm{II}}\right), 55.0(\mathrm{~d}$, $\left.{ }^{2} J_{\mathrm{CP}}=15.2, \mathrm{CHNH}\right), 92.5\left(\mathrm{~d},{ }^{1} J_{\mathrm{CP}}=206.7, \mathrm{PC}=\mathrm{C}\right), 125.6\left(\mathrm{C}_{6}\right), 126.9\left(\mathrm{C}_{2}\right), 127.9\left(\mathrm{C}_{4}\right), 131.0$ $\left(\mathrm{C}_{5}\right), 133.4\left(\mathrm{C}_{3}\right), 147.3\left(\mathrm{C}_{1}\right), 149.9\left(\mathrm{~d},{ }^{2} \mathrm{~J}_{\mathrm{CP}}=20.5, \mathrm{PC}=\mathrm{C}\right), 152.8(\mathrm{C}=\mathrm{O}) ;{ }^{1} \mathrm{H}$ NMR $\left(\mathrm{DMSO}-\mathrm{d}_{6}\right)$ $\delta 2.09\left(\mathrm{~d}, J_{\mathrm{HP}}=2.4,3 \mathrm{H}, \mathrm{CCH}_{3}\right), 3.36\left(\mathrm{~d},{ }^{3} \mathrm{~J}_{\mathrm{HP}}=11.4,3 \mathrm{H}, \mathrm{CH}_{3} \mathrm{O}^{\mathrm{I}}\right), 3.44\left(\mathrm{~d},{ }^{3} J_{\mathrm{HP}}=11.3,3 \mathrm{H}\right.$, $\left.\mathrm{CH}_{3} \mathrm{O}^{\mathrm{II}}\right), 4.87\left(\mathrm{dd},{ }^{3} J_{\mathrm{HP}}=8.6, J_{\mathrm{HH}}=3.5,1 \mathrm{H}, \mathrm{CHNH}\right), 7.23-7.31(\mathrm{~m}, 2 \mathrm{H}, \mathrm{ArH}), 7.32-7.42$ $(\mathrm{m}, 2 \mathrm{H}, \mathrm{ArH}), 7.74\left(\mathrm{~d}, J_{\mathrm{HH}}=2.0,1 \mathrm{H}, \mathrm{NH}\right), 9.27\left(\mathrm{~d}, J_{\mathrm{HH}}=2.7,1 \mathrm{H}, \mathrm{NH}\right) ;[\mathrm{M}+\mathrm{H}]^{+}{ }_{\text {found }}=$ 331.0617, $[\mathrm{M}+\mathrm{H}]^{+}$calculated $=331.0614$.

5-Dimethoxyphosphoryl-6-methyl-4-(4-chlorophenyl)-3,4-dihydropyrimidin-2(1H)-one (13d): Yield: $67 \%$ (0.22 g), white solid; Mp: $226-227{ }^{\circ} \mathrm{C}$; Mp [27]: 226-227 ${ }^{\circ} \mathrm{C} ;{ }^{31} \mathrm{P}\left(\mathrm{DMSO}-\mathrm{d}_{6}\right) \delta 22.8 ;{ }^{31} \mathrm{P}$ $\left(\mathrm{DMSO}-\mathrm{d}_{6}\right) \delta[27] 22.6 ;[\mathrm{M}+\mathrm{H}]^{+}$found $=331.0613,[\mathrm{M}+\mathrm{H}]^{+}$calculated $=331.0614$.

5-Dimethoxyphosphoryl-6-methyl-4-(4-fluorophenyl)-3,4-dihydropyrimidin-2(1H)-one (13e): Yield: $81 \%$ (0.25 g), white solid; Mp: $234-235{ }^{\circ} \mathrm{C}$; Mp [28]: $232-233{ }^{\circ} \mathrm{C} ;{ }^{31} \mathrm{P}\left(\mathrm{DMSO}-\mathrm{d}_{6}\right) \delta 22.7 ;{ }^{13} \mathrm{C}$ NMR $\left(\mathrm{DMSO}_{-} \mathrm{d}_{6}\right) \delta 17.8\left(\mathrm{~d},{ }^{3} J_{\mathrm{CP}}=3.6, \mathrm{CCH}_{3}\right), 51.9\left(\mathrm{~d},{ }^{2} J_{\mathrm{CP}}=5.0, \mathrm{CH}_{3} \mathrm{O}^{\mathrm{I}}\right), 52.0\left(\mathrm{~d},{ }^{2} J_{\mathrm{CP}}=5.3\right.$, $\left.\mathrm{CH}_{3} \mathrm{O}^{\mathrm{II}}\right), 54.8\left(\mathrm{~d},{ }^{2} J_{\mathrm{CP}}=15.3, \mathrm{CHNH}\right), 93.0\left(\mathrm{~d},{ }^{1} J_{\mathrm{CP}}=206.2, \mathrm{PC}=\mathrm{C}\right), 115.6\left(\mathrm{~d},{ }^{2} J_{\mathrm{CF}}=21.3, \mathrm{C}_{3}\right)$, $129.0\left(\mathrm{~d},{ }^{3} J_{\mathrm{CF}}=8.0, \mathrm{C}_{2}\right), 141.3\left(\mathrm{~d}, J_{\mathrm{CF}}=3.0, \mathrm{C}_{1}\right), 149.4\left(\mathrm{~d},{ }^{2} J_{\mathrm{CP}}=20.6, \mathrm{PC}=C\right), 152.8(\mathrm{C}=\mathrm{O})$, $161.9\left(\mathrm{~d},{ }^{1} J_{\mathrm{CF}}=243.2, \mathrm{C}_{4}\right) ;{ }^{1} \mathrm{H}$ NMR $\left(\mathrm{DMSO}_{-} \mathrm{d}_{6}\right) \delta 2.09\left(\mathrm{~d}, J_{\mathrm{HP}}=2.4,3 \mathrm{H}, \mathrm{CCH}_{3}\right), 3.32(\mathrm{~d}$, $\left.{ }^{3} J_{\mathrm{HP}}=11.4,3 \mathrm{H}, \mathrm{CH}_{3} \mathrm{O}^{\mathrm{I}}\right), 3.42\left(\mathrm{~d},{ }^{3} J_{\mathrm{HP}}=11.4,3 \mathrm{H}, \mathrm{CH}_{3} \mathrm{O}^{\mathrm{II}}\right), 4.85\left(\mathrm{dd},{ }^{3} J_{\mathrm{HP}}=8.6, J_{\mathrm{HH}}=3.4\right.$, 1H, CHNH), 7.12-7.20 (m, 2H, ArH), 7.27-7.33 (m, 2H, ArH), 7.68 (br s, 1H, NH), 9.21 $\left(\mathrm{d}, J_{\mathrm{HH}}=2.7,1 \mathrm{H}, \mathrm{NH}\right) ;{ }^{1} \mathrm{H} \mathrm{NMR}\left(\mathrm{CDCl}_{3}+\mathrm{DMSO}_{6}\right) \delta[28] 2.11\left(\mathrm{~s}, 3 \mathrm{H}, \mathrm{CCH}_{3}\right), 3.34(\mathrm{~d}$, $\left.{ }^{3} J_{\mathrm{HP}}=11.2,3 \mathrm{H}, \mathrm{CH}_{3} \mathrm{O}^{\mathrm{I}}\right), 3.39\left(\mathrm{~d},{ }^{3} J_{\mathrm{HP}}=11.3,3 \mathrm{H}, \mathrm{CH}_{3} \mathrm{O}^{\mathrm{II}}\right), 4.90\left(\mathrm{dd},{ }^{3} J_{\mathrm{HP}}=8.5, J_{\mathrm{HH}}=3.2\right.$, 1H, CHNH), 7.02-7.09 (m, 2H, ArH), 7.31-7.35 (m, 2H, ArH), 7.63 (s, 1H, NH), 9.17 (d, $\left.J_{\mathrm{HH}}=3.4,1 \mathrm{H}, \mathrm{NH}\right) ;[\mathrm{M}+\mathrm{H}]^{+}$found $=315.0910,[\mathrm{M}+\mathrm{H}]^{+}$calculated $=315.0909$.

5-Dimethoxyphosphoryl-6-methyl-4-(2-fluoro-4-iodophenyl)-3,4-dihydropyrimidin-2(1H)-one (13f): Yield: $63 \%$ (0.28 g), white solid; Mp: $250-251{ }^{\circ} \mathrm{C} ;{ }^{31} \mathrm{P}\left(\mathrm{DMSO}-\mathrm{d}_{6}\right) \delta 22.2 ;{ }^{13} \mathrm{C}$ NMR (DMSO$\left.\mathrm{d}_{6}\right) \delta 17.8\left(\mathrm{~d},{ }^{3} J_{\mathrm{CP}}=3.5, \mathrm{CCH}_{3}\right), 49.5\left(\mathrm{dd},{ }^{2} J_{\mathrm{CP}}=14.9,{ }^{3} J_{\mathrm{CF}}=2.9, \mathrm{CHNH}\right), 51.8\left(\mathrm{~d},{ }^{2} J_{\mathrm{CP}}=5.1\right.$, $\left.\mathrm{CH}_{3} \mathrm{O}^{\mathrm{I}}\right), 52.0\left(\mathrm{~d},{ }^{2} J_{\mathrm{CP}}=5.3, \mathrm{CH}_{3} \mathrm{O}^{\mathrm{II}}\right), 90.7\left(\mathrm{~d},{ }^{1} J_{\mathrm{CP}}=207.5, \mathrm{PC}=\mathrm{C}\right), 94.0\left(\mathrm{~d},{ }^{3} J_{\mathrm{CF}}=8.2, \mathrm{C}_{6}\right)$, $124.7\left(\mathrm{~d},{ }^{2} J_{\mathrm{CF}}=24.7, \mathrm{C}_{3}\right), 131.1\left(\mathrm{~d},{ }^{3} J_{\mathrm{CF}}=4.1, \mathrm{C}_{5}\right), 131.6\left(\mathrm{~d},{ }^{2} J_{\mathrm{CF}}=13.9, \mathrm{C}_{1}\right), 134.0(\mathrm{~d}$, $\left.{ }^{3} J_{\mathrm{CF}}=3.1, \mathrm{C}_{4}\right), 150.2\left(\mathrm{~d},{ }^{2} J_{\mathrm{CP}}=21.1, \mathrm{PC}=\mathrm{C}\right), 152.3(\mathrm{C}=\mathrm{O}), 159.4\left(\mathrm{~d},{ }^{1} J_{\mathrm{CF}}=252.6, \mathrm{C}_{2}\right) ;{ }^{1} \mathrm{H}$ NMR $\left(\mathrm{DMSO}_{-} \mathrm{d}_{6}\right) \delta 2.11\left(\mathrm{~d}, J_{\mathrm{HP}}=2.4,3 \mathrm{H}, \mathrm{CCH}_{3}\right), 3.27\left(\mathrm{~d},{ }^{3} J_{\mathrm{HP}}=11.2,3 \mathrm{H}, \mathrm{CH}_{3} \mathrm{O}^{\mathrm{I}}\right), 3.44(\mathrm{~d}$, $\left.{ }^{3} J_{\mathrm{HP}}=11.3,3 \mathrm{H}, \mathrm{CH}_{3} \mathrm{O}^{\mathrm{II}}\right), 5.11\left(\mathrm{dd},{ }^{3} \mathrm{~J}_{\mathrm{HP}}=8.0, J_{\mathrm{HH}}=3.2,1 \mathrm{H}, \mathrm{CHNH}\right), 7.03\left(\mathrm{t}, J_{\mathrm{HH}}=8.0,1 \mathrm{H}\right.$, $\mathrm{ArH}), 7.48-7.77$ [7.67 (br s, NH) overlapped by the multiplet of ArH total int. 3H], $9.26(\mathrm{~d}$, $\left.J_{\mathrm{HH}}=2.8,1 \mathrm{H}, \mathrm{NH}\right) ;[\mathrm{M}+\mathrm{H}]^{+}$found $=440.9870,[\mathrm{M}+\mathrm{H}]^{+}$calculated $=440.9876$.

5-Dimethoxyphosphoryl-6-methyl-4-(3-methylphenyl)-3,4-dihydropyrimidin-2(1H)-one (13g) Yield: $76 \%$ (0.24 g), white solid; Mp: $205-206{ }^{\circ} \mathrm{C} ;{ }^{31} \mathrm{P}\left(\mathrm{DMSO}_{6}\right) \delta 22.9 ;{ }^{13} \mathrm{C}$ NMR $\left(\mathrm{DMSO}-\mathrm{d}_{6}\right)$ $\delta 17.9\left(\mathrm{~d},{ }^{3} J_{\mathrm{CP}}=3.5, \mathrm{CCH}_{3}\right), 21.7\left(\mathrm{C}_{3} \mathrm{CH}_{3}\right), 51.8\left(\mathrm{~d},{ }^{2} J_{\mathrm{CP}}=5.0, \mathrm{CH}_{3} \mathrm{O}^{\mathrm{I}}\right), 52.0\left(\mathrm{~d},{ }^{2} J_{\mathrm{CP}}=5.3\right.$, $\left.\mathrm{CH}_{3} \mathrm{O}^{\mathrm{II}}\right), 55.4\left(\mathrm{~d},{ }^{2} J_{\mathrm{CP}}=15.1, \mathrm{CHNH}\right), 93.0\left(\mathrm{~d},{ }^{1} J_{\mathrm{CP}}=205.8, \mathrm{PC}=\mathrm{C}\right), 124.1\left(\mathrm{C}_{6}\right), 127.6\left(\mathrm{C}_{2}\right)$, 
$128.6\left(\mathrm{C}_{4}\right), 128.8\left(\mathrm{C}_{5}\right), 137.9\left(\mathrm{C}_{3}\right), 144.9\left(\mathrm{C}_{1}\right), 149.3\left(\mathrm{~d},{ }^{2} J_{\mathrm{CP}}=20.8, \mathrm{PC}=\mathrm{C}\right), 153.0(\mathrm{C}=\mathrm{O}) ;{ }^{1} \mathrm{H}$ NMR $\left(D_{M S O} d_{6}\right) \delta 2.09\left(\mathrm{~d}, J_{\mathrm{HP}}=2.5,3 \mathrm{H}, \mathrm{CCH}_{3}\right), 2.29\left(\mathrm{~s}, 3 \mathrm{H}, \mathrm{C}_{3} \mathrm{CH}_{3}\right), 3.30\left(\mathrm{~d},{ }^{3} \mathrm{~J}_{\mathrm{HP}}=11.2\right.$, $\left.3 \mathrm{H}, \mathrm{CH}_{3} \mathrm{O}^{\mathrm{I}}\right), 3.42\left(\mathrm{~d},{ }^{3} J_{\mathrm{HP}}=11.5,3 \mathrm{H}, \mathrm{CH}_{3} \mathrm{O}^{\mathrm{II}}\right), 4.80\left(\mathrm{dd},{ }^{3} J_{\mathrm{HP}}=8.8, J_{\mathrm{HH}}=3.5,1 \mathrm{H}, \mathrm{CHNH}\right)$, 7.04-7.11 (m, 3H, ArH), 7.18-7.25 (m, 1H, ArH), $7.64\left(\mathrm{~d}, 1 \mathrm{H}, J_{\mathrm{HH}}=2.2, \mathrm{NH}\right), 9.16(\mathrm{~d}$, $\left.J_{\mathrm{HH}}=2.7,1 \mathrm{H}, \mathrm{NH}\right) ;[\mathrm{M}+\mathrm{H}]^{+}$found $=311.1160,[\mathrm{M}+\mathrm{H}]^{+}$calculated $=311.1160$.

5-Dimethoxyphosphoryl-6-methyl-4-(4-hydroxyphenyl)-3,4-dihydropyrimidin-2(1H)-one (13h): Yield: $60 \%$ (0.19 g), white solid; Mp: $230-231{ }^{\circ} \mathrm{C} ;{ }^{31} \mathrm{P}\left(\right.$ DMSO- $\left._{6}\right) \delta 23.2 ;{ }^{13} \mathrm{C}$ NMR (DMSO$\left.\mathrm{d}_{6}\right) \delta 17.8\left(\mathrm{~d},{ }^{3} J_{\mathrm{CP}}=3.6, \mathrm{CCH}_{3}\right), 51.8\left(\mathrm{~d},{ }^{2} J_{\mathrm{CP}}=5.0, \mathrm{CH}_{3} \mathrm{O}^{\mathrm{I}}\right), 51.9\left(\mathrm{~d},{ }^{2} J_{\mathrm{CP}}=5.2, \mathrm{CH}_{3} \mathrm{O}^{\mathrm{II}}\right), 54.9$ $\left(\mathrm{d},{ }^{2} J_{\mathrm{CP}}=15.3, \mathrm{CHNH}\right), 93.5\left(\mathrm{~d},{ }^{1} J_{\mathrm{CP}}=205.3, \mathrm{PC}=\mathrm{C}\right), 115.5\left(\mathrm{C}_{3}\right), 128.2\left(\mathrm{C}_{2}\right), 135.6\left(\mathrm{C}_{1}\right), 148.8$ $\left(\mathrm{d},{ }^{2} J_{\mathrm{CP}}=20.8, \mathrm{PC}=\mathrm{C}\right), 153.0(\mathrm{C}=\mathrm{O}), 157.2\left(\mathrm{C}_{4}\right) ;{ }^{1} \mathrm{H}$ NMR $\left(\mathrm{DMSO}-\mathrm{d}_{6}\right) \delta 2.08\left(\mathrm{~d}, J_{\mathrm{HP}}=2.5\right.$, $\left.3 \mathrm{H}, \mathrm{CCH}_{3}\right), 3.28\left(\mathrm{~d},{ }^{3} J_{\mathrm{HP}}=11.2,3 \mathrm{H}, \mathrm{CH}_{3} \mathrm{O}^{\mathrm{I}}\right), 3.40\left(\mathrm{~d},{ }^{3} J_{\mathrm{HP}}=11.3,3 \mathrm{H}, \mathrm{CH}_{3} \mathrm{O}^{\mathrm{II}}\right), 4.72(\mathrm{dd}$, $\left.{ }^{3} J_{\mathrm{HP}}=8.7, J_{\mathrm{HH}}=3.4,1 \mathrm{H}, \mathrm{CHNH}\right), 6.61-6.81(\mathrm{~m}, 2 \mathrm{H}, \mathrm{ArH}), 6.99-7.12(\mathrm{~m}, 2 \mathrm{H}, \mathrm{ArH}), 7.54(\mathrm{br}$ $\mathrm{s}, 1 \mathrm{H}, \mathrm{NH}), 9.10\left(\mathrm{~d}, J_{\mathrm{HH}}=2.9,1 \mathrm{H}, \mathrm{NH}\right), 9.34(\mathrm{~s}, 1 \mathrm{H}, \mathrm{OH}) ;[\mathrm{M}+\mathrm{H}]^{+}$found $=313.0956,[\mathrm{M}+$ $\mathrm{H}^{+}$calculated $=313.0953$.

5-Dimethoxyphosphoryl-6-methyl-4-(4-nitrophenyl)-3,4-dihydropyrimidin-2(1H)-one (13i): Yield: $68 \%$ (0.23 g), yellow solid; Mp: $235-236{ }^{\circ} \mathrm{C}$; Mp [27]: $235-237{ }^{\circ} \mathrm{C} ;{ }^{31} \mathrm{P}\left(\mathrm{DMSO}^{\mathrm{d}} \mathrm{d}_{6}\right) \delta 22.3 ;{ }^{31} \mathrm{P}$ $\left(\mathrm{DMSO}_{-} \mathrm{d}_{6}\right) \delta[27] 22.3 ;[\mathrm{M}+\mathrm{H}]^{+}$found $=342.0856,\left[\mathrm{M}+\mathrm{H}^{+}{ }_{\text {calculated }}=342.0854\right.$.

5-Dimethoxyphosphoryl-6-methyl-4-(3,4,5-trimethoxyphenyl)-3,4-dihydropyrimidin-2(1H)-one (13j): Yield: $56 \%$ (0.22 g), white solid; Mp: $219-220{ }^{\circ} \mathrm{C} ;{ }^{31} \mathrm{P}\left(\mathrm{DMSO}_{\mathrm{d}}\right) \delta 23.0 ;{ }^{13} \mathrm{C}$ NMR (DMSO$\left.\mathrm{d}_{6}\right) \delta 17.8\left(\mathrm{~d},{ }^{3} J_{\mathrm{CP}}=3.6, \mathrm{CCH}_{3}\right), 52.0\left(\mathrm{~d},{ }^{2} J_{\mathrm{CP}}=4.7, \mathrm{CH}_{3} \mathrm{O}^{\mathrm{I}}\right), 52.1\left(\mathrm{~d},{ }^{2} J_{\mathrm{CP}}=5.5, \mathrm{CH}_{3} \mathrm{O}^{\mathrm{II}}\right)$, $55.1\left(\mathrm{~d},{ }^{2} J_{\mathrm{CP}}=15.1, \mathrm{CHNH}\right), 56.3\left(\mathrm{C}_{3} \mathrm{OCH}_{3}\right), 60.5\left(\mathrm{C}_{4} \mathrm{OCH}_{3}\right), 96.8\left(\mathrm{~d},{ }^{1} J_{\mathrm{CP}}=206.6, \mathrm{PC}=\mathrm{C}\right)$, 104.4 $\left(\mathrm{C}_{2}\right), 137.4\left(\mathrm{C}_{3}\right), 140.3\left(\mathrm{C}_{4}\right), 149.6\left(\mathrm{~d},{ }^{2} J_{\mathrm{CP}}=20.6, \mathrm{PC}=\mathrm{C}\right), 153.0(\mathrm{C}=\mathrm{O}), 153.2\left(\mathrm{C}_{1}\right) ;{ }^{1} \mathrm{H}$ NMR $\left(\right.$ DMSO- $\left.\mathrm{d}_{6}\right) \delta 2.06\left(\mathrm{~d}, J_{\mathrm{HP}}=2.4,3 \mathrm{H}, \mathrm{CCH}_{3}\right), 3.37\left(\mathrm{~d},{ }^{3} J_{\mathrm{HP}}=11.2,3 \mathrm{H}, \mathrm{CH}_{3} \mathrm{O}^{\mathrm{I}}\right), 3.46(\mathrm{~d}$, $\left.{ }^{3} J_{\mathrm{PH}}=11.3,3 \mathrm{H}, \mathrm{CH}_{3} \mathrm{O}^{\mathrm{II}}\right), 3.61\left(\mathrm{~s}, 3 \mathrm{H}, \mathrm{C}_{4} \mathrm{OCH}_{3}\right), 3.71\left(\mathrm{~s}, 6 \mathrm{H}, \mathrm{C}_{3} \mathrm{OCH}_{3}\right), 4.80\left(\mathrm{dd},{ }^{3} J_{\mathrm{HP}}=9.0\right.$, $\left.J_{\mathrm{HH}}=3.4,1 \mathrm{H}, \mathrm{CHNH}\right), 6.58(\mathrm{~s}, 2 \mathrm{H}, \mathrm{ArH}), 7.64(\mathrm{br} \mathrm{s}, 1 \mathrm{H}, \mathrm{NH}), 9.16\left(\mathrm{~d}, J_{\mathrm{HH}}=5.5,1 \mathrm{H}, \mathrm{NH}\right)$; $[\mathrm{M}+\mathrm{H}]^{+}$found $=387.1322,[\mathrm{M}+\mathrm{H}]^{+}$calculated $=387.1321$.

5-Diethoxyphosphoryl-1,6-dimethyl-4-phenyl-3,4-dihydropyrimidin-2(1H)-one (14a): Yield: 56\% (0.19 g), light yellow oil; ${ }^{31} \mathrm{P}\left(\right.$ DMSO-d $\left._{6}\right) \delta 19.9,20.9 ;{ }^{13} \mathrm{C}$ NMR $\left(\right.$ DMSO-d $\left._{6}\right) \delta 16.3\left(\mathrm{~d}_{\text {, }}\right.$ $\left.{ }^{3} J_{\mathrm{CP}}=6.8, \mathrm{CH}_{3} \mathrm{CH}_{2} \mathrm{O}^{\mathrm{I}}\right), 16.5\left(\mathrm{~d},{ }^{3} J_{\mathrm{CP}}=6.5, \mathrm{CH}_{3} \mathrm{CH}_{2} \mathrm{O}^{\mathrm{II}}\right), 17.6\left(\mathrm{~d},{ }^{3} J_{\mathrm{CP}}=3.7, \mathrm{CCH}_{3}\right), 29.2$ $\left(\mathrm{NCH}_{3}\right), 53.7\left(\mathrm{~d},{ }^{2} J_{\mathrm{CP}}=14.1, \mathrm{CHNH}\right), 61.16\left(\mathrm{~d},{ }^{3} J_{\mathrm{CP}}=4.1, \mathrm{CH}_{3} \mathrm{CH}_{2} \mathrm{O}^{\mathrm{I}}\right), 61.23\left(\mathrm{~d},{ }^{3} J_{\mathrm{CP}}=4.9\right.$, $\left.\mathrm{CH}_{3} \mathrm{CH}_{2} \mathrm{O}^{\mathrm{II}}\right), 97.3\left(\mathrm{~d},{ }^{1} J_{\mathrm{CP}}=203.4, \mathrm{PC}=\mathrm{C}\right), 126.9\left(\mathrm{C}_{3}\right), 127.9\left(\mathrm{C}_{4}\right), 128.8\left(\mathrm{C}_{2}\right), 144.2(\mathrm{~d}$, $\left.{ }^{3} J_{\mathrm{CP}}=1.5, \mathrm{C}_{1}\right), 151.1\left(\mathrm{~d},{ }^{2} J_{\mathrm{CP}}=21.5, \mathrm{PC}=\mathrm{C}\right), 153.9\left(\mathrm{~d}, J_{\mathrm{CP}}=1.5, \mathrm{C}=\mathrm{O}\right) ;{ }^{1} \mathrm{H}$ NMR $\left(\mathrm{DMSO}-\mathrm{d}_{6}\right)$ $\delta 1.00\left(\mathrm{t}, J_{\mathrm{HH}}=7.1,3 \mathrm{H}, \mathrm{CH}_{3} \mathrm{CH}_{2} \mathrm{O}^{\mathrm{I}}\right), 1.15\left(\mathrm{t}, J_{\mathrm{HH}}=7.0,3 \mathrm{H}, \mathrm{CH}_{3} \mathrm{CH}_{2} \mathrm{O}^{\mathrm{II}}\right), 2.32\left(\mathrm{~d}, J_{\mathrm{HP}}=2.5\right.$, $\left.3 \mathrm{H}, \mathrm{CCH}_{3}\right), 3.08\left(\mathrm{~s}, 3 \mathrm{H}, \mathrm{NCH}_{3}\right), 3.51-3.68\left(\mathrm{~m}, 1 \mathrm{H}, \mathrm{CH}_{\mathrm{A}}, \mathrm{CH}_{3} \mathrm{CH}_{2} \mathrm{O}^{\mathrm{I}}\right), 3.71-3.91(\mathrm{~m}, 3 \mathrm{H}$, $\left.\mathrm{CH}_{\mathrm{B}}, \mathrm{CH}_{3} \mathrm{CH}_{2} \mathrm{O}^{\mathrm{I}}, \mathrm{CH}_{3} \mathrm{CH}_{2} \mathrm{O}^{\mathrm{II}}\right), 4.84\left(\mathrm{dd},{ }^{3} J_{\mathrm{HP}}=10.1, J_{\mathrm{HH}}=3.9,1 \mathrm{H}, \mathrm{CHNH}\right), 7.17-7.42,(\mathrm{~m}$, $5 \mathrm{H}, \mathrm{ArH}) 7.85(\mathrm{br} \mathrm{s}, 1 \mathrm{H}, \mathrm{NH}) ;[\mathrm{M}+\mathrm{H}]^{+}$found $=339.1473,[\mathrm{M}+\mathrm{H}]^{+}$calculated $=339.1474$.

5-Dimethoxyphosphoryl-1,6-dimethyl-4-phenyl-3,4-dihydropyrimidin-2(1H)-one (14b): Yield: $53 \%$ (0.16 g), white solid; Mp: $127-128{ }^{\circ} \mathrm{C} ;{ }^{31} \mathrm{P}\left(\mathrm{DMSO}^{\mathrm{d}} \mathrm{d}_{6}\right) \delta 23.1,23.9 ;{ }^{13} \mathrm{C}$ NMR (DMSO$\left.\mathrm{d}_{6}\right) \delta 17.6\left(\mathrm{~d},{ }^{3} J_{\mathrm{CP}}=3.7, \mathrm{CCH}_{3}\right), 30.0\left(\mathrm{NCH}_{3}\right), 52.0\left(\mathrm{~d}^{2}{ }^{2} J_{\mathrm{CP}}=5.0, \mathrm{CH}_{3} \mathrm{O}^{\mathrm{I}}\right), 52.2\left(\mathrm{~d},{ }^{2} J_{\mathrm{CP}}=5.4\right.$, $\left.\mathrm{CH}_{3} \mathrm{O}^{\mathrm{II}}\right), 53.6\left(\mathrm{~d},{ }^{2} J_{\mathrm{CP}}=14.3, \mathrm{CHNH}\right), 96.0\left(\mathrm{~d},{ }^{1} J_{\mathrm{CP}}=204.4, \mathrm{PC}=\mathrm{C}\right), 126.9\left(\mathrm{C}_{3}\right), 127.9\left(\mathrm{C}_{4}\right)$, $128.89\left(\mathrm{C}_{2}\right), 144.2\left(\mathrm{~d},{ }^{3} J_{\mathrm{CP}}=1.4, \mathrm{C}_{1}\right), 151.7\left(\mathrm{~d},{ }^{2} J_{\mathrm{CP}}=21.4, \mathrm{PC}=\mathrm{C}\right), 153.9\left(\mathrm{~d}, J_{\mathrm{CP}}=1.5\right.$, $\mathrm{C}=\mathrm{O}) ;{ }^{1} \mathrm{H}$ NMR $\left(\mathrm{DMSO}_{6}\right) \delta 2.31\left(\mathrm{~d}, \mathrm{~J}_{\mathrm{HP}}=2.6,3 \mathrm{H}, \mathrm{CCH}_{3}\right), 3.08\left(\mathrm{~s}, 3 \mathrm{H}, \mathrm{NCH}_{3}\right), 3.36(\mathrm{~d}$, $\left.{ }^{3} J_{\mathrm{HP}}=11.2,3 \mathrm{H}, \mathrm{CH}_{3} \mathrm{O}^{\mathrm{I}}\right), 3.46\left(\mathrm{~d},{ }^{3} J_{\mathrm{HP}}=11.4,3 \mathrm{H}, \mathrm{CH}_{3} \mathrm{O}^{\mathrm{II}}\right), 4.82\left(\mathrm{dd},{ }^{3} J_{\mathrm{HP}}=10.1, J_{\mathrm{HH}}=3.9\right.$, $1 \mathrm{H}, \mathrm{CHNH}), 7.21-7.41,(\mathrm{~m}, 5 \mathrm{H}, \mathrm{ArH}) 7.89\left(\mathrm{t}, J_{\mathrm{HH}}=3.9,1 \mathrm{H}, \mathrm{NH}\right) ;[\mathrm{M}+\mathrm{H}]^{+}$found $=311.1159$, $[\mathrm{M}+\mathrm{H}]^{+}$calculated $=311.1161$.

5-Diethoxyphosphoryl-6-methyl-4-propyl-3,4-dihydropyrimidin-2(1H)-one (15a): Yield: 41\% (0.12 g), yellow oil; ${ }^{31} \mathrm{P}\left(\mathrm{DMSO}_{6}\right) \delta 20.5 ;{ }^{13} \mathrm{C}$ NMR $\left(\mathrm{DMSO}_{6}\right) \delta 14.2\left(\mathrm{CH}_{2} \mathrm{CH}_{2} \mathrm{CH}_{3}\right)$, $16.60\left(\mathrm{~d},{ }^{3} J_{\mathrm{CP}}=6.6, \mathrm{CH}_{3} \mathrm{CH}_{2} \mathrm{O}^{\mathrm{I}}\right), 16.61\left(\mathrm{~d},{ }^{3} \mathrm{~J}_{\mathrm{CP}}=6.6, \mathrm{CH}_{3} \mathrm{CH}_{2} \mathrm{O}^{\mathrm{II}}\right), 17.3\left(\mathrm{CH}_{2} \mathrm{CH}_{2} \mathrm{CH}_{3}\right)$, $17.7\left(\mathrm{~d},{ }^{3} J_{\mathrm{CP}}=3.7, \mathrm{CCH}_{3}\right), 39.7\left(\mathrm{CH}_{2} \mathrm{CH}_{2} \mathrm{CH}_{3}\right), 51.4\left(\mathrm{~d},{ }^{2} J_{\mathrm{CP}}=16.4, \mathrm{CHNH}\right), 61.0(\mathrm{~d}$, $\left.{ }^{2} J_{\mathrm{CP}}=5.3, \mathrm{CH}_{3} \mathrm{CH}_{2} \mathrm{O}^{\mathrm{I}}\right), 61.1\left(\mathrm{~d},{ }^{2} J_{\mathrm{CP}}=5.3, \mathrm{CH}_{3} \mathrm{CH}_{2} \mathrm{O}^{\mathrm{II}}\right), 94.3\left(\mathrm{~d},{ }^{1} J_{\mathrm{CP}}=204.2, \mathrm{PC}=\mathrm{C}\right), 148.5$ $\left(\mathrm{d},{ }^{2} J_{\mathrm{CP}}=21.2, \mathrm{PC}=C\right), 153.6\left(\mathrm{~d}, J_{\mathrm{CP}}=1.3, \mathrm{C}=\mathrm{O}\right) ;{ }^{1} \mathrm{H}$ NMR $\left(\mathrm{DMSO}-\mathrm{d}_{6}\right) \delta 0.86\left(\mathrm{t}, J_{\mathrm{HH}}=6.7\right.$, $\left.3 \mathrm{H}, \mathrm{CH}_{2} \mathrm{CH}_{2} \mathrm{CH}_{3}\right), 1.12-1.53\left[1.22\left(\mathrm{t}, J_{\mathrm{HH}}=7.0, \mathrm{CH}_{3} \mathrm{CH}_{2} \mathrm{O}\right)\right.$ overlapped by the multiplet of 
$\mathrm{CH}_{2} \mathrm{CH}_{2} \mathrm{CH}_{3}, \mathrm{CH}_{2} \mathrm{CH}_{2} \mathrm{CH}_{3}$ total int. 10H], $2.00\left(\mathrm{~d}, J_{\mathrm{HP}}=2.6,3 \mathrm{H}, \mathrm{CCH}_{3}\right), 3.71-3.82(\mathrm{~m}, 1 \mathrm{H}$, $\left.\mathrm{CH}_{\mathrm{A}}, \mathrm{CH}_{3} \mathrm{CH}_{2} \mathrm{O}^{\mathrm{I}}\right), 3.84-4.00\left(\mathrm{~m}, 4 \mathrm{H}, \mathrm{CH}_{\mathrm{B}}, \mathrm{CH}_{3} \mathrm{CH}_{2} \mathrm{O}^{\mathrm{I}}, \mathrm{CH}_{3} \mathrm{CH}_{2} \mathrm{O}^{\mathrm{II}}, \mathrm{CHNH}\right), 7.25$ (br s, $1 \mathrm{H}$, $\mathrm{NH}), 8.85\left(\mathrm{~d}, J_{\mathrm{HH}}=3.2,1 \mathrm{H}, \mathrm{NH}\right) ;[\mathrm{M}+\mathrm{H}]^{+}$found $=291.1472,[\mathrm{M}+\mathrm{H}]^{+}$calculated $=291.1473$.

5-Dimethoxyphosphoryl-6-methyl-4-propyl-3,4-dihydropyrimidin-2(1H)-one (15b): Yield: 43\% (0.11 g), yellow oil; ${ }^{31} \mathrm{P}\left(\mathrm{DMSO}_{6}\right) \delta 23.6 ;{ }^{13} \mathrm{C}$ NMR $\left(\mathrm{DMSO}_{6} \mathrm{~d}_{6}\right) \delta 14.2\left(\mathrm{CH}_{2} \mathrm{CH}_{2} \mathrm{CH}_{3}\right), 17.3$ $\left(\mathrm{CH}_{2} \mathrm{CH}_{2} \mathrm{CH}_{3}\right), 17.7\left(\mathrm{~d},{ }^{3} J_{\mathrm{CP}}=3.7, \mathrm{CCH}_{3}\right), 39.7\left(\mathrm{CH}_{2} \mathrm{CH}_{2} \mathrm{CH}_{3}\right), 51.4\left(\mathrm{~d},{ }^{2} J_{\mathrm{CP}}=14.7, \mathrm{CHNH}\right)$, $52.0\left(\mathrm{~d}^{2} J_{\mathrm{CP}}=5.2, \mathrm{CH}_{3} \mathrm{O}^{\mathrm{I}}\right), 52.1\left(\mathrm{~d},{ }^{2} J_{\mathrm{CP}}=5.4, \mathrm{CH}_{3} \mathrm{O}^{\mathrm{II}}\right), 93.0\left(\mathrm{~d},{ }^{1} J_{\mathrm{CP}}=204.6, \mathrm{PC}=\mathrm{C}\right), 149.2$ $\left(\mathrm{d},{ }^{2} J_{\mathrm{CP}}=21.2, \mathrm{PC}=\mathrm{C}\right), 153.6\left(\mathrm{~d}, J_{\mathrm{CP}}=1.3, \mathrm{C}=\mathrm{O}\right) ;{ }^{1} \mathrm{H}$ NMR $\left(\mathrm{DMSO} \mathrm{d}_{6}\right) \delta 0.85\left(\mathrm{t}, J_{\mathrm{HH}}=7.0\right.$, $\left.3 \mathrm{H}, \mathrm{CH}_{2} \mathrm{CH}_{2} \mathrm{CH}_{3}\right), 1.16-1.26\left(\mathrm{~m}, 1 \mathrm{H}, \mathrm{CH}_{2} \mathrm{CH}_{2} \mathrm{CH}_{3}, \mathrm{CH}_{\mathrm{A}}\right), 1.30-1.46\left(\mathrm{~m}, 3 \mathrm{H}, \mathrm{CH}_{2} \mathrm{CH}_{2} \mathrm{CH}_{3}\right.$, $\left.\mathrm{CH}_{\mathrm{B}}, \mathrm{CH}_{2} \mathrm{CH}_{2} \mathrm{CH}_{3}\right), 2.00\left(\mathrm{~d}, \mathrm{~J}_{\mathrm{HP}}=2.4,3 \mathrm{H}, \mathrm{CCH}_{3}\right), 3.71-3.82\left(\mathrm{~m}, 1 \mathrm{H}, \mathrm{CH}_{\mathrm{A}}, \mathrm{CH}_{3} \mathrm{CH}_{2} \mathrm{O}^{\mathrm{I}}\right)$, $3.57\left(\mathrm{~d},{ }^{3} J_{\mathrm{HP}}=11.3,3 \mathrm{H}, \mathrm{CH}_{3} \mathrm{O}^{\mathrm{I}}\right), 3.57\left(\mathrm{~d},{ }^{3} J_{\mathrm{HP}}=11.2,3 \mathrm{H}, \mathrm{CH}_{3} \mathrm{O}^{\mathrm{II}}\right), 43.71-3.79(\mathrm{~m}, 1 \mathrm{H}$, $\mathrm{CHNH}), 7.26-7.30(\mathrm{~m}, 1 \mathrm{H}, \mathrm{NH}), 8.92\left(\mathrm{~d}, \mathrm{~J}_{\mathrm{HH}}=2.8,1 \mathrm{H}, \mathrm{NH}\right) ;[\mathrm{M}+\mathrm{H}]^{+}$found $=263.1156,[\mathrm{M}+$ $\mathrm{H}^{+}{ }_{\text {calculated }}=263.1160$.

5-Diethoxyphosphoryl-6-methyl-4-isobutyl-3,4-dihydropyrimidin-2(1H)-one (16a): Yield: 43\% $(0.13 \mathrm{~g})$, yellow oil; ${ }^{31} \mathrm{P}\left(\mathrm{DMSO}_{6}\right) \delta 20.2 ;{ }^{13} \mathrm{C}$ NMR $\left(\mathrm{DMSO}_{6} \mathrm{~d}_{6}\right) \delta 16.2\left(\mathrm{~d},{ }^{3} J_{\mathrm{CP}}=6.4\right.$, $\left.\mathrm{CH}_{3} \mathrm{CH}_{2} \mathrm{O}\right), 17.3\left(\mathrm{~d},{ }^{3} J_{\mathrm{CP}}=3.5, \mathrm{CCH}_{3}\right), 21.1\left(\mathrm{CH}_{2} \mathrm{CHCH}_{3}{ }^{\mathrm{I}}\right), 22.7\left(\mathrm{CH}_{2} \mathrm{CHCH}_{3}{ }^{\mathrm{II}}\right), 23.9$ $\left(\mathrm{CH}_{2} \mathrm{CH}\left(\mathrm{CH}_{3}\right)_{2}\right), 45.6\left(\mathrm{CH}_{2} \mathrm{CH}\left(\mathrm{CH}_{3}\right)_{2}\right), 49.1\left(\mathrm{~d},{ }^{2} J_{\mathrm{CP}}=14.1, \mathrm{CHNH}\right), 60.5\left(\mathrm{~d},{ }^{2} J_{\mathrm{CP}}=5.3\right.$, $\left.\mathrm{CH}_{3} \mathrm{CH}_{2} \mathrm{O}^{\mathrm{I}}\right), 60.7\left(\mathrm{~d},{ }^{2} J_{\mathrm{CP}}=5.4, \mathrm{CH}_{3} \mathrm{CH}_{2} \mathrm{O}^{\mathrm{II}}\right), 95.0\left(\mathrm{~d},{ }^{1} J_{\mathrm{CP}}=204.5, \mathrm{PC}=\mathrm{C}\right), 147.9(\mathrm{~d}$, $\left.{ }^{2} J_{\mathrm{CP}}=21.7, \mathrm{PC}=\mathrm{C}\right), 153.2(\mathrm{C}=\mathrm{O}) ;{ }^{1} \mathrm{H}$ NMR $\left(\mathrm{DMSO}^{\mathrm{d}} \mathrm{d}\right) \delta 0.84\left(\mathrm{~d}, \mathrm{~J}_{\mathrm{HH}}=6.5,3 \mathrm{H},\left(\mathrm{CH}_{2} \mathrm{CHCH}_{3} \mathrm{I}^{\mathrm{I}}\right)\right.$, $0.86\left(\mathrm{~d}, J_{\mathrm{HH}}=6.7,3 \mathrm{H},\left(\mathrm{CH}_{2} \mathrm{CHCH}_{3}{ }^{\mathrm{II}}\right), 1.08-1.15\left(\mathrm{~m}, 1 \mathrm{H}, \mathrm{CH}_{2} \mathrm{CH}\left(\mathrm{CH}_{3}\right)_{2}, \mathrm{CH}_{\mathrm{A}}\right), 1.22(\mathrm{t}\right.$, $\left.J_{\mathrm{HH}}=7.1,6 \mathrm{H}, \mathrm{CH}_{3} \mathrm{CH}_{2} \mathrm{O}\right), 1.39-1.46\left(\mathrm{~m}, 1 \mathrm{H}, \mathrm{CH}_{2} \mathrm{CH}\left(\mathrm{CH}_{3}\right)_{2}, \mathrm{CH}_{\mathrm{B}}\right), 1.68-1.77(\mathrm{~m}, 1 \mathrm{H}$, $\left.\mathrm{CH}_{2} \mathrm{CH}\left(\mathrm{CH}_{3}\right)_{2}\right), 2.01\left(\mathrm{~d}, J_{\mathrm{HP}}=2.4,3 \mathrm{H}, \mathrm{CCH}_{3}\right), 3.72-3.80(\mathrm{~m}, 1 \mathrm{H}, \mathrm{CHNH}), 3.87-3.98(\mathrm{~m}, 4 \mathrm{H}$, $\left.\mathrm{CH}_{3} \mathrm{CH}_{2} \mathrm{O}\right), 7.37-7.41(\mathrm{~m}, 1 \mathrm{H}, \mathrm{NH}), 8.88\left(\mathrm{~d}, \mathrm{~J}_{\mathrm{HH}}=4.8,1 \mathrm{H}, \mathrm{NH}\right) ;[\mathrm{M}+\mathrm{H}]^{+}$found $=305.1632$, $[\mathrm{M}+\mathrm{H}]^{+}$calculated $=305.1630$.

5-Dimethoxyphosphoryl-6-methyl-4-isobutyl-3,4-dihydropyrimidin-2(1H)-one (16b): Yield: 42\% (0.12 g), yellow oil ; ${ }^{31} \mathrm{P}\left(\mathrm{DMSO}_{6}\right) \delta 20.2 ;{ }^{13} \mathrm{C}$ NMR $\left(\mathrm{DMSO}_{6} \mathrm{~d}_{6}\right) \delta 17.3\left(\mathrm{~d},{ }^{3} J_{\mathrm{CP}}=3.6, \mathrm{CCH}_{3}\right)$, $21.6\left(\mathrm{CH}_{2} \mathrm{CHCH}_{3}{ }^{\mathrm{I}}\right), 23.2\left(\mathrm{CH}_{2} \mathrm{CHCH}_{3}{ }^{\mathrm{II}}\right), 24.3\left(\mathrm{CH}_{2} \mathrm{CH}\left(\mathrm{CH}_{3}\right)_{2}\right), 46.2\left(\mathrm{CH}_{2} \mathrm{CH}\left(\mathrm{CH}_{3}\right)_{2}\right), 49.5$ $\left(\mathrm{d},{ }^{2} J_{\mathrm{CP}}=14.3, \mathrm{CHNH}\right), 52.0\left(\mathrm{~d},{ }^{2} J_{\mathrm{CP}}=10.2, \mathrm{CH}_{3} \mathrm{O}^{\mathrm{I}}\right), 52.1\left(\mathrm{~d},{ }^{2} J_{\mathrm{CP}}=10.1, \mathrm{CH}_{3} \mathrm{O}^{\mathrm{II}}\right), 94.2$ $\left(\mathrm{d},{ }^{1} J_{\mathrm{CP}}=204.8, \mathrm{PC}=\mathrm{C}\right), 149.0\left(\mathrm{~d},{ }^{2} J_{\mathrm{CP}}=21.6, \mathrm{PC}=\mathrm{C}\right), 153.5\left(\mathrm{~d}, J_{\mathrm{CP}}=1.2, \mathrm{C}=\mathrm{O}\right) ;{ }^{1} \mathrm{H}$ NMR $\left(\mathrm{DMSO}_{-} \mathrm{d}_{6}\right) \delta 0.83\left(\mathrm{~d}, \mathrm{~J}_{\mathrm{HH}}=6.5,3 \mathrm{H},\left(\mathrm{CH}_{2} \mathrm{CHCH}_{3}{ }^{\mathrm{I}}\right), 0.86\left(\mathrm{~d}, \mathrm{~J}_{\mathrm{HH}}=6.8,3 \mathrm{H}, \mathrm{CH}_{2} \mathrm{CHCH}_{3}{ }^{\mathrm{II}}\right)\right.$, 1.02-1.18 (m, $\left.1 \mathrm{H}, \mathrm{CH}_{2} \mathrm{CH}\left(\mathrm{CH}_{3}\right)_{2}, \mathrm{CH}_{\mathrm{A}}\right), 1.37-1.49\left(\mathrm{~m}, 1 \mathrm{H}, \mathrm{CH}_{2} \mathrm{CH}\left(\mathrm{CH}_{3}\right)_{2}, \mathrm{CH}_{\mathrm{B}}\right), 1.64-1.80$ $\left(\mathrm{m}, 1 \mathrm{H}, \mathrm{CH}_{2} \mathrm{CH}\left(\mathrm{CH}_{3}\right)_{2}\right), 2.00\left(\mathrm{~d}, J_{\mathrm{HP}}=2.6,3 \mathrm{H}, \mathrm{CCH}_{3}\right), 3.57\left(\mathrm{~d},{ }^{3} J_{\mathrm{HP}}=11.3,3 \mathrm{H}, \mathrm{CH}_{3} \mathrm{O}^{\mathrm{I}}\right)$, $3.57\left(\mathrm{~d},{ }^{3} J_{\mathrm{PH}}=11.2,3 \mathrm{H}, \mathrm{CH}_{3} \mathrm{O}^{\mathrm{II}}\right), 3.68-3.80(\mathrm{~m}, 1 \mathrm{H}, \mathrm{CHNH}), 7.33-7.45(\mathrm{~m}, 1 \mathrm{H}, \mathrm{NH}), 8.93(\mathrm{~d}$, $\left.J_{\mathrm{HH}}=3.4,1 \mathrm{H}, \mathrm{NH}\right) ;[\mathrm{M}+\mathrm{H}]^{+}$found $=277.1317,[\mathrm{M}+\mathrm{H}]^{+}$calculated $=277.1317$.

3.5. General Procedure for the Synthesis of 5-Diethoxyphosphoryl-4-phenyl-6-styryl-3,4dihydropyrimidin-2(1H)-One (12a)

A mixture of $1.0 \mathrm{mmol}(0.32 \mathrm{~g})$ 5-diethoxyphosphoryl-6-methyl-4-phenyl-3,4dihydropyrimidin-2(1H)-one (11a), $2.0 \mathrm{mmol}(0.21 \mathrm{~mL})$ benzaldehyde and $0.10 \mathrm{mmol}$ $(0.036 \mathrm{~g})$ zinc triflate was heated in acetonitrile at $82{ }^{\circ} \mathrm{C}$ for $24 \mathrm{~h}$ in an oil bath. The volatile components were removed in vacuum, and the residue was purified by column chromatography using silica gel as the absorbent and dichloromethane:methanol (97:3) as the eluent. The following product was prepared:

5-Diethoxyphosphoryl-4-phenyl-6-styryl-3,4-dihydropyrimidin-2(1H)-one (12a): Yield: 25\% (0.10 g), white solid; Mp: 194-195 ${ }^{\circ} \mathrm{C} ;{ }^{31} \mathrm{P}\left(\right.$ DMSO- $\left._{6}\right) \delta 18.4 ;{ }^{13} \mathrm{C}$ NMR $($ DMSO-d 6 ) $\delta 16.2(\mathrm{~d}$, $\left.{ }^{3} J_{\mathrm{CP}}=6.7, \mathrm{CH}_{3} \mathrm{CH}_{2} \mathrm{O}^{\mathrm{I}}\right), 16.6\left(\mathrm{~d},{ }^{3} J_{\mathrm{CP}}=6.1, \mathrm{CH}_{3} \mathrm{CH}_{2} \mathrm{O}^{\mathrm{II}}\right), 55.6\left(\mathrm{~d},{ }^{2} J_{\mathrm{CP}}=13.4, \mathrm{CHNH}\right), 61.4$ $\left(\mathrm{d},{ }^{2} J_{\mathrm{CP}}=4.9, \mathrm{CH}_{3} \mathrm{CH}_{2} \mathrm{O}^{\mathrm{I}}\right), 61.5\left(\mathrm{~d},{ }^{2} J_{\mathrm{CP}}=5.0, \mathrm{CH}_{3} \mathrm{CH}_{2} \mathrm{O}^{\mathrm{II}}\right), 98.7\left(\mathrm{~d},{ }^{1} J_{\mathrm{CP}}=200.8, \mathrm{PC}=\mathrm{C}\right)$, $119.9\left(\mathrm{~d},{ }^{3} J_{\mathrm{CP}}=5.0, \mathrm{CH}=\mathrm{CHPh}\right), 127.1\left(\mathrm{C}_{3}\right), 127.4\left(\mathrm{C}_{3^{\prime}}\right), 128.1\left(\mathrm{C}_{4}\right), 129.0\left(\mathrm{C}_{2}\right), 129.49\left(\mathrm{C}_{4^{\prime}}\right)$, $129.52\left(\mathrm{C}_{2^{\prime}}\right), 133.8\left(\mathrm{C}_{1}\right), 136.4\left(\mathrm{C}_{1^{\prime}}\right), 144.4\left(\mathrm{~d}, J_{\mathrm{CP}}=1.5, \mathrm{CH}=\mathrm{CHPh}\right), 145.9\left(\mathrm{~d},{ }^{2} J_{\mathrm{CP}}=20.1\right.$, $\mathrm{PC}=\mathrm{C}), 153.3(\mathrm{C}=\mathrm{O}) ;{ }^{1} \mathrm{H}$ NMR $\left(\mathrm{DMSO}^{\mathrm{d}} \mathrm{d}\right) \delta 0.93\left(\mathrm{t}, \mathrm{J}_{\mathrm{HH}}=7.0,3 \mathrm{H}, \mathrm{CH}_{3} \mathrm{CH}_{2} \mathrm{O}^{\mathrm{I}}\right), 1.16(\mathrm{t}$, $\left.J_{\mathrm{HH}}=7.0,3 \mathrm{H}, \mathrm{CH}_{3} \mathrm{CH}_{2} \mathrm{O}^{\mathrm{II}}\right), 3.46-3.62\left(\mathrm{~m}, 1 \mathrm{H}, \mathrm{CH}_{\mathrm{A}}, \mathrm{CH}_{3} \mathrm{CH}_{2} \mathrm{O}^{\mathrm{I}}\right), 3.72-3.92\left(\mathrm{~m}, 3 \mathrm{H}, \mathrm{CH}_{\mathrm{B}}\right.$, $\left.\mathrm{CH}_{3} \mathrm{CH}_{2} \mathrm{O}^{\mathrm{I}}, \mathrm{CH}_{3} \mathrm{CH}_{2} \mathrm{O}^{\mathrm{II}}\right), 4.97\left(\mathrm{dd},{ }^{3} J_{\mathrm{HP}}=9.6, J_{\mathrm{HH}}=3.5,1 \mathrm{H}, \mathrm{CHNH}\right), 7.20-7.55(\mathrm{~m}, 11 \mathrm{H}$, 
$\mathrm{ArH}, \mathrm{CH}=\mathrm{CH}), 7.56-7.69(\mathrm{~m}, 1 \mathrm{H}, \mathrm{CH}=\mathrm{CH}), 7.79(\mathrm{br} \mathrm{s}, 1 \mathrm{H}, \mathrm{NH}), 9.23\left(\mathrm{~d}, J_{\mathrm{HH}}=3.5,1 \mathrm{H}, \mathrm{NH}\right)$; $[\mathrm{M}+\mathrm{H}]^{+}$found $=413.1631,[\mathrm{M}+\mathrm{H}]^{+}$calculated $=413.1630$.

\section{Conclusions}

In this paper, the synthesis of novel 3,4-dihydropyrimidin-2(1H)-one-phosphonates was elaborated by the solvent-free MW-assisted three-component condensation of $\beta$ ketophosphonates, aromatic or aliphatic aldehydes and urea derivatives. The Biginelli reaction was optimized in respect of the heating method, the type of the catalyst and solvent, the temperature, the reaction time, and the molar ratio of the starting materials. The optimized conditions were found to be 1.5 equivalents of benzaldehyde and 2 equivalents of urea in the presence of $15 \mathrm{~mol} \%$ of $\mathrm{Zn}(\mathrm{OTf})_{2}$ at $100{ }^{\circ} \mathrm{C}$ for $2 \mathrm{~h}$ under solvent-free MW irradiation. The MW-assisted method developed was then extended to a range of aromatic aldehydes, $\beta$-ketophosphonates and urea derivatives to prove the wide scope and functional group tolerance of the process. The 5-diethoxyphosphoryl-4phenyl-6-styryl-3,4-dihydropyrimidin-2(1H)-one, as a new by-product of the condensation, was isolated and characterized. Our MW-assisted approach made also possible the Biginelli reaction of aliphatic aldehydes, diethyl (2-oxopropyl)phosphonate and urea, which transformation was previously reported as impossible in the literature. Altogether 26 3,4-dihydropyrimidin-2(1H)-one-phosphonates of which 20 derivatives are new, were synthesized in yields of $41-81 \%$.

Supplementary Materials: Supplementary data associated with this article are available online at https:/ / www.mdpi.com/2073-4344/11/1/45/s1. Copies of ${ }^{31} \mathrm{P},{ }^{1} \mathrm{H}$, and ${ }^{13} \mathrm{C}$ NMR spectra for all compounds synthesized are presented.

Author Contributions: E.B., N.P.-T. and Á.T. planned the experiments, N.P.-T. and E.H. carried out the experiments, E.B. contributed reagents/materials/analysis tools. E.B., N.P.-T. and Á.T. All authors have read and agreed to the published version of the manuscript.

Funding: The project was supported by the Hungarian Research Development and Innovation Office (FK123961). N.P.-T. was supported by the Servier-Beregi PhD Research Fellowship. E.B. was supported by the János Bolyai Research Scholarship of the Hungarian Academy of Sciences (BO/00278/17/7) and by the ÚNKP-20-5-BME-288 New National Excellence Program of the Ministry of Human Capacities.

Data Availability Statement: Data is contained within the article or Supplementary Materials. The data presented in this study are available in https://www.mdpi.com/2073-4344/11/1/45/s1.

Conflicts of Interest: The authors declare no conflict of interest.

\section{References}

1. Allen, D.W.; Loakes, D.; Tebby, J.C. SPR—Organophosphorus Chemistry; Royal Society of Chemistry: Cambridge, UK, 2016; Volume 45.

2. Allen, M.C.; Fuhrer, W.; Tuck, B.; Wade, R. Renin inhibitors. Synthesis of transition-state analog inhibitors containing phosphorus acid derivatives at the scissile bond. J. Med. Chem. 1989, 32, 1652-1661. [CrossRef]

3. Kafarski, P.; Lejczak, B. Aminophosphonic acids of potential medical importance. Curr. Med. Chem. Anticancer. Agents 2001, 1, 301-312. [CrossRef]

4. Mucha, A.; Kafarski, P.; Berlicki, L. Remarkable potential of the $\alpha$-aminophosphonate/phosphinate structural motif in medicinal chemistry. J. Med. Chem. 2011, 54, 5955-5980. [CrossRef]

5. Atherton, F.R.; Hassal, C.H.; Lambert, R.W. Synthesis and structure-activity relationships of antibacterial phosphonopeptides incorporating (1-aminoethyl)phosphonic acid and (aminomethyl)phosphonic acid. J. Med. Chem. 1986, 29, 29-40. [CrossRef]

6. Fields, S.C. Synthesis of natural products containing a C-P bond. Tetrahedron 1999, 55, 12237-12272. [CrossRef]

7. Haji, M. Multicomponent Reactions: A simple and efficient route to heterocyclic phosphonates. Beilstein J. Org. Chem. 2016, 12, 1269-1301. [CrossRef]

8. Orru, R.V.A.; Rujiter, E. Phosphorus Heterocycles II. In Topics in Heterocyclic Chemistry; Bansal, R.K., Ed.; Springer: Berlin, Germany, 2010; Volume 21.

9. Jiang, Z.; Zhao, J.; Gao, B.; Chen, S.; Qu, W.; Mei, X.; Rui, C.; Ning, J.; She, D. Synthesis and application of N-tosyl piperidinylcontaining $\alpha$-aminophosphonates. Phosphorus Sulfur Silicon Relat. Elem. 2013, 188, 1026-1037. [CrossRef] 
10. Tripolszky, A.; Tóth, E.; Szabó, P.T.; Hackler, L.; Kari, B.; Puskás, L.G.; Bálint, E. Synthesis and in vitro cytotoxicity and antibacterial activity of novel 1,2,3-triazol-5-yl-phosphonates. Molecules 2020, 25, 2643. [CrossRef]

11. Morita, I.; Tada, S.I.; Kunimoto, K.; Tsuda, M.; Kiese, M.; Kimura, K. Synthesis and antihypertensive activities of 1,4dihydropyridine-5-phosphonate derivatives. I. Chem. Pharm. Bull. 1987, 35, 3898-3904. [CrossRef]

12. Morita, I.; Tada, S.I.; Kunimoto, K.; Tsuda, M.; Kiese, M.; Kimura, K. Synthesis and antihypertensive activities of 1,4dihydropyridine-5-phosphonate derivatives. II. Chem. Pharm. Bull. 1987, 35, 4144-4154. [CrossRef]

13. Morita, I.; Tada, S.I.; Kunimoto, K.; Tsuda, M.; Kiese, M.; Kimura, K. Synthesis and antihypertensive activities of 1,4dihydropyridine-5-phosphonate derivatives. III. Chem. Pharm. Bull. 1987, 35, 4819-4828. [CrossRef] [PubMed]

14. Sandhu, S.; Sandhu, J.S. Past, present and future of the Biginelli reaction: A critical perspective. ARKIVOC 2012, 1, 66-133.

15. Daur, R.; Chaudhary, S.; Kumar, K.; Gupta, M.K.; Rawal, R.K. Recent synthetic and medicinal perspectives of dihydropyrimidinones: A review. Eur. J. Med. Chem. 2017, 132, 108-134.

16. Mayer, T.U.; Kapoor, T.M.; Haggarty, S.J.; King, R.W.; Schreiber, S.L.; Mitchison, T.J. Small molecule inhibitor of mitotic spindle bipolarity identified in a phenotype-based screen. Science 1999, 286, 971-974. [CrossRef]

17. Russowsky, D.; Canto, R.F.S.; Sanches, S.A.A.; D'Oca, M.G.M.; de Fátima, A.; Pilli, R.A.; Kohn, L.K.; Antônio, M.A.; de Carvalho, J.E. Synthesis and differential antiproliferative activity of Biginelli compounds against cancer cell lines: Monastrol, oxo-monastrol and oxygenated analogues. Bioorg. Chem. 2006, 34, 173-182. [CrossRef]

18. Essid, I.; Lahbib, K.; Kaminsky, W.; Nasr, C.B.; Touil, S. 5-Phosphonato-3,4-dihydropyrimidin-2(1H)-ones: Zinc triflate-catalyzed onepot multi-component synthesis, X-ray crystal structure and anti-inflammatory activity. J. Mol. Struct. 2017, 1142, 130-138. [CrossRef]

19. Zhu, J.; Wang, Q.; Wang, M.X. Multicomponent Reactions in Organic Synthesis; Wiley: Wienheim, Germany, 2015.

20. Cioc, R.C.; Ruijter, E.; Orru, R.V.A. Multicomponent reactions: Advanced tools for sustainable organic synthesis. Green Chem. 2014, 16, 2958-2975. [CrossRef]

21. Jiang, B.; Shi, F.; Tu, S.J. Microwave-assisted multicomponent reactions in the heterocyclic chemistry. Curr. Org. Chem. 2010, 14, 357-378. [CrossRef]

22. Kruithof, A.; Ruijter, E.; Orru, R.V.A. Microwave-assisted multicomponent reactions in the synthesis of heterocycles. In Microwaves in Organic Synthesis, 3rd ed.; de la Hoz, A., Loupy, A., Eds.; Wiley: Weinheim, Germany, 2012; Volume 2, pp. $1099-1171$.

23. Bálint, E.; Keglevich, G. The spread of the application of the microwave technique in organic synthesis. In Milestones in Microwave Chemistry; Keglevich, G., Ed.; Springer: Cham, Switzerland, 2016; pp. 1-10.

24. Kappe, C.O.; Stadler, A.; Dallinger, D. Microwaves in Organic and Medicinal Chemistry; Wiley: New Jersey, NJ, USA, $2012 ;$ Volume 25.

25. Kappe, C.O.; Stadler, A. The Biginelli dihydropyrimidine synthesis. In Organic Reactions; Overman, L.E., Ed.; Wiley: Hoboken, NJ, USA, 2004; Volume 63, pp. 1-116.

26. Mohammadi, M.; Behbahani, F.K. Recent developments in the synthesis and applications of dihydropyrimidin-2(1H)-ones and thiones. Mol. Divers. 2018, 22, 405-446. [CrossRef]

27. Essid, I.; Touil, S. $\beta$-Ketophosphonates as substrates in the Biginelli multicomponent reaction: An efficient and straightforward synthesis of phosphorylated dihydropyrimidinones. ARKIVOC 2013, 4, 98-106. [CrossRef]

28. Gong, D.; Zhang, L.; Yuan, C. A convenient synthesis of 5-(O,O-dialkylphosphoryl)-4-aryl-3,4-dihydropyrimidin-2(1H)-ones. Heteroatom Chem. 2003, 14, 13-17. [CrossRef]

29. Chebil, E.; Touil, S. Behaviour of $\beta-$ keto- $\delta$-carbethoxyphosphonates and phosphine oxides in the Biginelli multicomponent reaction: Regioselective synthesis of 5-carbethoxy-6-phosphonomethyl-3,4-dihydropyrimidin-2-ones. Heterocycles 2012, 85, 2765-2774.

30. Zhang, L.; Zhang, Z.; Liu, Q.; Liu, T.; Zhang, G. Iron-catalyzed vinylogous aldol condensation of Biginelli products and its application toward pyrido[4,3-d]pyrimidinones. J. Org. Chem. 2014, 79, 2281-2288. [CrossRef] 